\title{
Nanoparticle O-H Bond Dissociation Free Energies from Equilibrium Measurements of Cerium Oxide Colloids
}

\author{
Rishi G. Agarwal, Hyun-Jo Kim, and James M. Mayer*† \\ †Department of Chemistry, Yale University, New Haven, CT 06520-8107, USA \\ *Corresponding author, james.mayer@yale.edu
}

\section{Table of Contents}

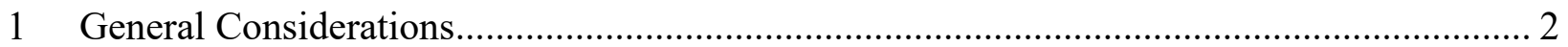

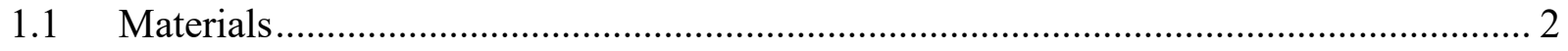

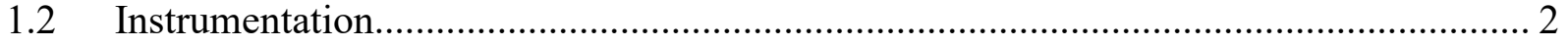

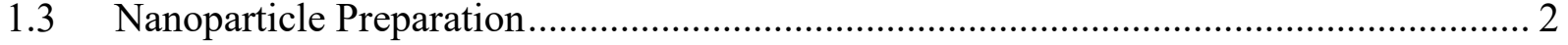

$1.4 \quad{ }^{1} \mathrm{H}$ NMR Spectroscopy Time Course Experimental Details ............................................. 3

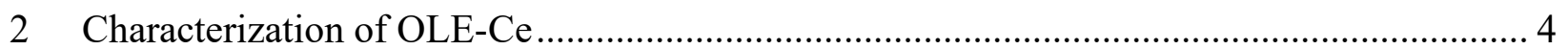

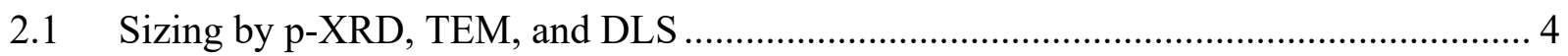

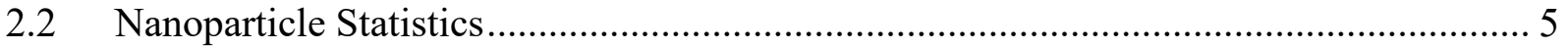

2.3 ICP-MS Determinations of the Concentration of Cerium Atoms in OLE-Ce Colloids... 7

$2.4{ }^{1} \mathrm{H}$ NMR Determination of the Concentration of Oleate/Oleic Acid Capping Ligands... 8

3 X-ray Absorption Spectroscopy Experimental Details ………............................................ 8

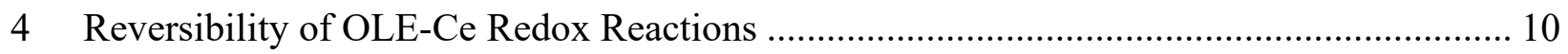

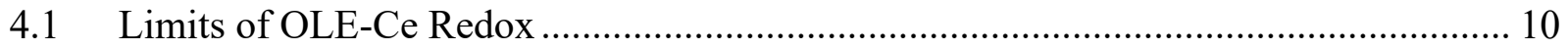

4.2 Linear Correlations between Molar Absorptivity and $\% \mathrm{Ce}^{3+}$ for OLE-Ce Colloids ..... 12

4.3 Redox Effects on OLE-Ce Size and Capping Ligands .................................................. 13

5 Synthesis and Characterization of Organic PCET Reagents ……..................................... 15

6 Establishing Equilibrium between OLE-Ce and PCET Reagents in THF ............................ 17

6.1 ${ }^{1} \mathrm{H}$ NMR spectra for Equilibrium of OLE-Ce and PCET Reductants ............................. 17

$6.2{ }^{1} \mathrm{H}$ NMR spectra for Equilibrium of OLE-Ce and PCET Oxidants ............................... 22

6.3 Compiled ${ }^{1} \mathrm{H}$ NMR and XAS Data for Equilibrations ................................................ 27

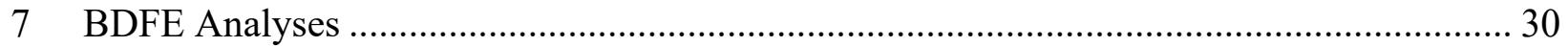

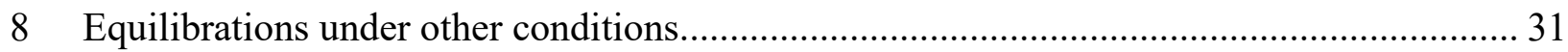

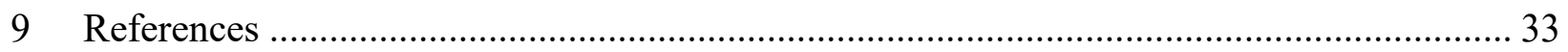




\section{General Considerations}

\section{$1.1 \quad$ Materials}

All chemicals were purchased commercially (vendor given as mentioned) and used without further purification, unless otherwise specified. 1,3,5-trimethoxybenzene (TMB) was purchased from SigmaAldrich. See section 5 for details on the purification of quinones and synthesis of hydroquinones used in this study. All deuterated solvents were purchased from Cambridge Isotope Laboratories, degassed, and dried over sieves for $>2$ days before use. All proteo solvents used were treated using a Pure Process Technology solvent purification system which degassed solvent with Argon and dried it over activated alumina, unless otherwise specified. Tetrahydrofuran (THF) was always inhibitor-free (Fisher, HPLC grade). All water was dispensed from a Synergy ${ }^{\circledR}-\mathrm{R}$ Millipore system as ultra-pure water $(18.2 \mathrm{M} \Omega \cdot \mathrm{cm})$ unless otherwise specified.

\subsection{Instrumentation}

Powder X-ray diffraction (p-XRD) patterns were obtained on Rigaku MiniFlex600 Powder X-Ray Diffractometer with sealed $\mathrm{Cu}$ X-ray source $(\lambda=1.5418 \AA) .{ }^{1} \mathrm{H}$ NMR spectra for reactions were always collected on an Agilent DD2 $500 \mathrm{MHz}$ spectrometer, while those for characterization were also carried out on an Agilent DD2 $400 \mathrm{MHz}$ spectrometer and an Agilent DD2 $600 \mathrm{MHz}$ spectrometer. Data were processed using MestReNova ${ }^{\circ}$ software. Chemical shifts were reported relative to TMS by referencing the residual proteo solvent in THF- $\mathrm{d}_{8}$ solutions or a 1,3,5-trimethoxybenzene internal standard for THF/THF$\mathrm{d}_{8}$ mixtures. Dynamic Light Scattering (DLS) data was collected on a NanoBrook Omni, Brookhaven Instruments using a $640 \mathrm{~nm}$ laser. Transmission Electron Microscopy (TEM) images were obtained on FEI Tecnai Osiris $200 \mathrm{kV}$ transmission electron microscope operating at 200kV. TEM samples were prepared on the bench by dropping $10 \mu \mathrm{L}$ of a [Ce atoms] $=1 \mathrm{mM}$ solution in cyclohexane (Sigma-Aldrich, Spectroscopy grade, used from bottle) on a lacey carbon 400 mesh $\mathrm{Cu}$ grid (Ted Pella, Inc.) and allowing the grid to air-dry. The size and the distribution of NCs were obtained by counting nanoparticles with ImageJ software. Inductively-coupled plasma mass spectroscopy (ICP-MS) was carried out using a Perkin Elmer ICP-MS Elan DRC-e instrument with Fluka TraceCERT ${ }^{\circledR}$ High Purity brand Ce standard $(1000 \pm 2$ $\mathrm{mg} / \mathrm{L}$ in $2 \% \mathrm{HCl}$ ). UV-Vis data were collected using an Agilent Cary $60 \mathrm{UV}-\mathrm{V}$ is spectrophotometer.

\subsection{Nanoparticle Preparation}

Oleate-capped ceria NCs (OLE-Ce) were synthesized through modification of a published procedure. ${ }^{1}$ Sodium oleate $\left(\mathrm{C}_{17} \mathrm{H}_{33} \mathrm{COONa}\right.$, STREM, 99\%, $2.12 \mathrm{~g}$, $\left.7.0 \mathrm{mmol}\right)$ was dissolved in $15 \mathrm{~mL}$ of warmed ultrapure water $(18.2 \mathrm{M} \Omega \cdot \mathrm{cm})$. The resulting solution was then allowed to cool to room temperature before dropwise addition to a stirring solution of $1.92 \mathrm{~g}(3.5 \mathrm{mmol})$ ceric ammonium nitrate $\left(\left(\mathrm{NH}_{4}\right)_{2} \mathrm{Ce}\left(\mathrm{NO}_{3}\right)_{6}\right.$, Sigma-Aldrich, $99.9 \%$ ) in $15 \mathrm{~mL}$ ultra-pure water to give a fine white precipitate. Subsequently, $4.5 \mathrm{~mL}$ of aqueous ammonia solution (28 wt. \% ( $\geq 99.99 \%$ trace metals basis), Sigma-Aldrich) was added dropwise under vigorous stirring to the reaction mixture over the course of $\sim 1$ minute. After $\sim 10$ minutes a homogeneous light tan colloid formed. The mixture was stirred for an additional 45 minutes. Products were collected by centrifugation ( $6500 \mathrm{rpm}$ for $15 \mathrm{~min}$.), followed by decantation, two washes with ultra-pure water to remove excess starting materials, and two washes with ethanol to remove excess oleic acid. After the last centrifugation and decantation, the remaining tan solids were consolidated into two glass test tubes and dried at $90{ }^{\circ} \mathrm{C}$ for $24 \mathrm{~h}$ in an oven under air. The resulting black glassy solid was brought into an $\mathrm{N}_{2}$ glovebox, and dissolved in tetrahydrofuran, although a small amount of precipitate remained. These 
precipitates were removed by filtration. The resulting clear brown solution was stored under $\mathrm{N}_{2}$ in the freezer at $-30{ }^{\circ} \mathrm{C}$ (Figure S1).

Two batches of oleate-capped cerium oxide nanoparticles (Ce-1 and Ce-2) were prepared using the procedure above. A third batch $(\mathbf{C e}-\mathbf{L})$ was synthesized following the same procedure except that the tan colloid formed after addition of $\mathrm{NH}_{3}$ (aq) solution was heated to $150{ }^{\circ} \mathrm{C}$ for 21 hours in a Parr bomb reactor while stirring. ${ }^{1}$ The subsequent washing procedure was modified so that the solids were washed three times with ultra-pure water.

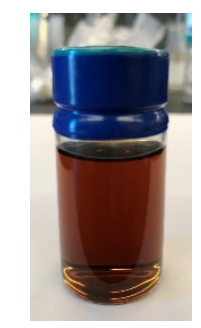

Figure S1. Picture of Ce-1 colloid in THF.

\section{4 ${ }^{1}$ H NMR Spectroscopy Time Course Experimental Details}

Quantitative ${ }^{1} \mathrm{H}$ NMR measurements were performed in mixtures of THF- $\mathrm{d}_{8}$ and proteo-THF with the aid of solvent suppression (PreSat pulse sequence) using 1,3,5-trimethoxybenzene (TMB) as an internal standard. Samples were measured in the presence of proteo-THF in order to avoid the extra manipulation involved in creating a stock of the nanoceria colloid in THF- $\mathrm{d}_{8}$. No significant differences were observed between measurements run in mixtures as opposed to only THF- $\mathrm{d}_{8}$. NMR Experiments used 16 scans and a relaxation delay of 40 seconds ( 5 times the highest $T_{1}$ for an integrated peak) to ensure proper quantitation. For stoichiometric and equilibration reactions with oleate-capped cerium oxide nanoparticles, the concentration of Ce atoms was generally $9 \mathrm{mM}$ and $8 \mathrm{mM}$ TMB was present. In equilibration reactions, an excess of the organic PCET reagent was always added ( $\sim 10 \mathrm{mM}$ of hydrogen atom equivalents).

All samples were prepared in NMR tubes equipped with J. Young valves using standard glovebox procedures in an air-free $\mathrm{N}_{2}$ environment at room temperature. For time courses, samples were stored in the glovebox and covered with aluminum foil between measurements. These steps were necessary to prevent $\mathrm{O}_{2}$ leakage through the J. Young seal and the light induced reaction of THF and quinones over the long timescales of experiments. ${ }^{2}$ Spectra were referenced to the aromatic peak of TMB, whose chemical shift $(6.043 \mathrm{ppm})$ was identified from experiments in THF- $\mathrm{d}_{8}$. MestReNova ${ }^{\circledR}$ was used to phase, baseline correct, and integrate spectra. Reactions were nearly complete in two days, but demonstrated multi-exponential kinetics. As a result, they were followed for over 1-2 weeks to ensure thermal equilibrium had been reached. Reactions were considered complete when the quantity of product had plateaued by ${ }^{1} \mathrm{H}$ NMR. Values of organic product produced were determined by averaging all time points in the plateau region. 


\section{Characterization of OLE-Ce}

\subsection{Sizing by $p-X R D, T E M$, and $D L S$}

Sizing of OLE-Ce colloids was evaluated by up to three methods. TEM (

Figure S2) gave the size of the inorganic core of the nanoparticle and standard deviation over a relatively small sample size (>200 nanoparticles), p-XRD (Figure S3) gave the size of crystalline domains for the bulk sample via the Scherrer equation, and DLS (Figure S4) probed the solution dispersity and hydrodynamic radius of OLE-Ce. The values determined from these three methods were self-consistent for Ce-2. The size determined from p-XRD is the smallest at $1.4 \pm 0.3 \mathrm{~nm}$ while the values from TEM is slightly larger at $1.9 \pm 0.3 \mathrm{~nm}$ consistent with an amorphous surface and crystalline core as TEM measures the size of the entire inorganic core, while p-XRD only measures the size of the crystalline domains. Additionally, sizing by DLS gives a significantly larger value of $4.7 \pm 1.0 \mathrm{~nm}$ because it should measure the size of the entire nanoparticle including ligands and associated solvent. Given that an unkinked structure of oleate is $\sim 2.1 \mathrm{~nm}$ this data is consistent with a non-aggregated solution nanoparticle structure.
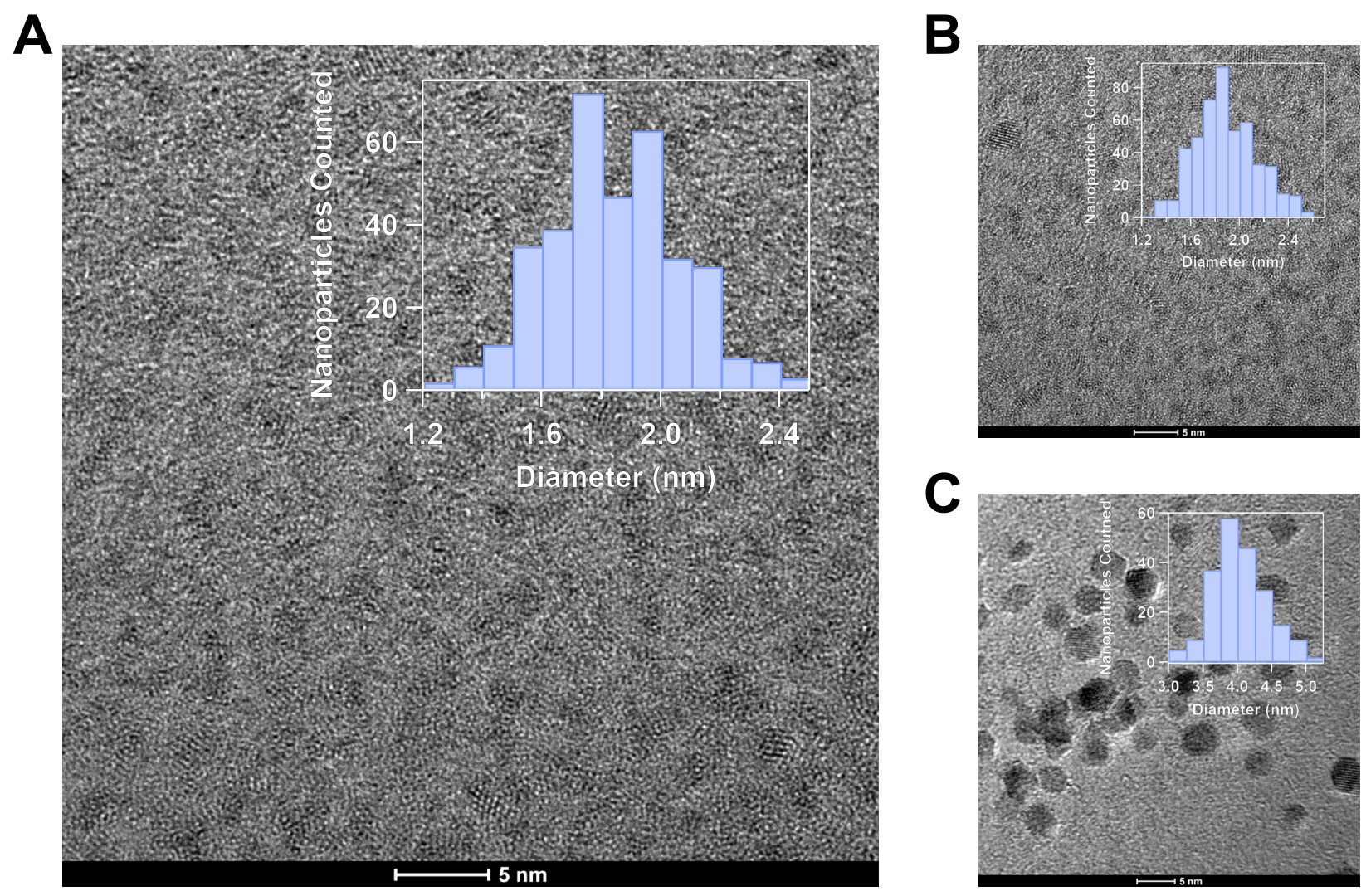

Figure S2. TEM images with size histograms of (A) Ce-1 with an avg. diameter of $1.8 \pm 0.2 \mathrm{~nm}$, (B) Ce-2 with an avg. diameter of $1.9 \pm 0.3 \mathrm{~nm}$, and (C) Ce-L with an avg. diameter of $4.0 \pm 0.4$ nm. 


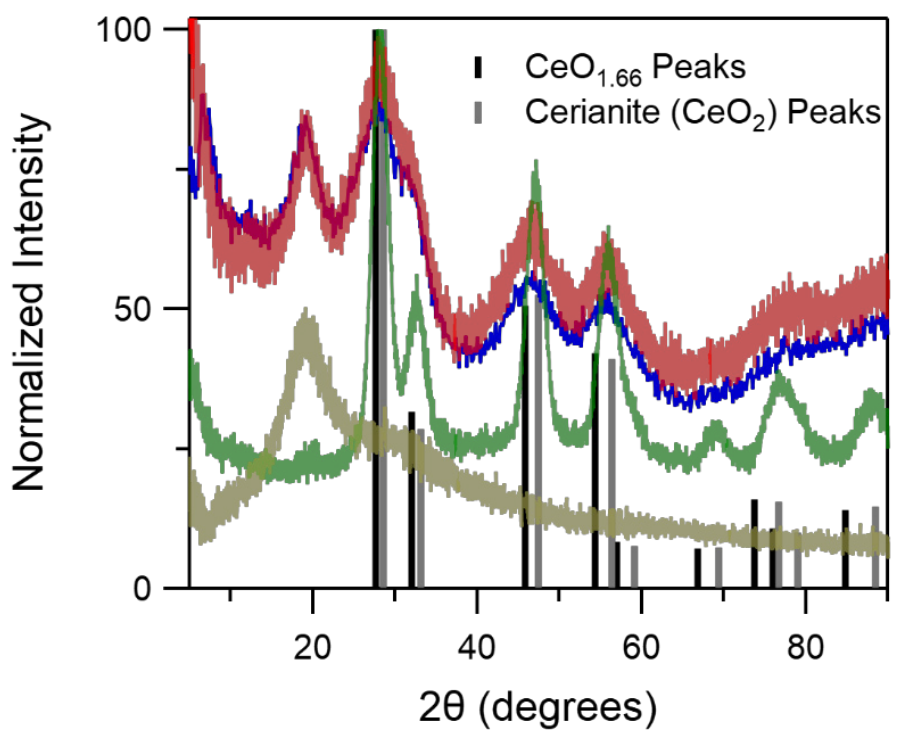

Figure S3. Powder XRD data of Ce-1 (blue), Ce-2 (red), Ce-L (green), and oleic acid (gold). Peak positions are best fit by cerianite ( $\mathrm{CeO}_{2}$, $\mathrm{COD}$ ID: 9009008$)$ and $\mathrm{CeO}_{1.66}$ (COD ID: 1521459). Samples were prepared on glass slides on the benchtop by concentrating OLE-Ce stock solutions with a hot plate. Estimated sizes are $1.3 \pm 0.3 \mathrm{~nm}, 1.4 \pm 0.3 \mathrm{~nm}$, and $3.2 \pm 0.5 \mathrm{~nm}$ for Ce-1, Ce-2, and Ce-L, respectively. Peak fits and size estimations were performed using Rigaku PDXL software.

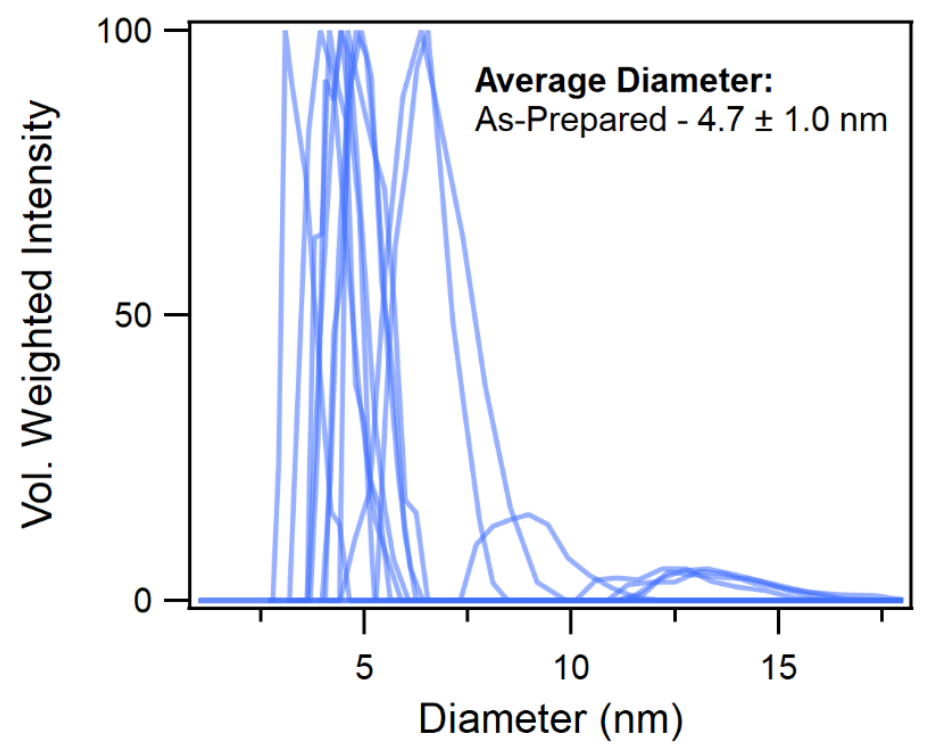

Figure S4. DLS data of Ce-2 One data set consists of ten runs and all are shown above. The average diameter was calculated to be $4.7 \pm 1.0 \mathrm{~nm}$ by averaging the diameter for the peak of Volume (Vol.) Weighted Intensity in each run. Calculation of the Vol. Weighted Intensity was achieved with Brookhaven Particle Solutions software. Samples were measured under air in glass cuvettes.

\subsection{Nanoparticle Statistics}

Calculation of cerium atoms per nanoparticle: 
The lattice parameter $(a)$ for fluorite unit cell of cerium oxide is $0.5411 \mathrm{~nm}$. We note that previous investigations have shown that the lattice parameter of cerium oxide nanoparticles is size dependent, but that this correction is smaller than the errors associated with this analysis. ${ }^{3}$

The volume of a nanoparticle $\left(V_{\mathrm{NP}}\right)$ is $4 / 3 \pi r^{3}$ calculated assuming a sphere of radius $r$, and the volume of the unit cell $\left(V_{\mathrm{uc}}\right)$ is $a^{3}$ for a fluorite structure. Therefore, since there are 4 Cerium atoms per unit cell, the number of cerium atoms per nanoparticle $\left(N_{\mathrm{NP}}\right)$ is calculated as follows:

$$
N_{\mathrm{NP}}=4 *\left(V_{\mathrm{NP}} / V_{\mathrm{uc}}\right)
$$

The number of surface cerium atoms per nanoparticle was estimated using two methods:

\section{Method 1:}

The surface is estimated as a symmetric truncated octahedron with 6 square (100) and 8 hexagonal (111) facets, as has been previously observed for uncapped ceria nanoparticles $(3-10 \mathrm{~nm})$ in this size regime. ${ }^{4}$ Under these assumptions the nanoparticle surface is $77.6 \%$ (111) and $22.4 \%$ (100) by area. Based upon the fluorite structure of the cerium oxide unit cell there are 1.875 cerium atoms per unit cell area of (111) facet and 0.875 cerium atoms per unit cell area of (100) facet. Furthermore, since cerium oxide adopts a fluorite structure the areas $(A)$ of the (111) and (100) facets is as follows:

$$
\begin{gathered}
A_{(111)}=\sqrt{ } 3 / 2 * a^{2} \\
A_{(100)}=a^{2}
\end{gathered}
$$

The number of surface cerium atoms per nanoparticle ( $\left.N_{\text {surf }}\right)$ is shown in eq $\mathrm{S} 4$ where the total surface area is estimated as that of a sphere $\left(4 \pi r^{2}\right)$.

$$
N_{\text {surf }}=1.875 \frac{0.776\left(4 \Pi r^{2}\right)}{A_{(111)}}+0.875 \frac{0.224\left(4 \Pi r^{2}\right)}{A_{(100)}}
$$

\section{Method 2:}

The number of surface cerium atoms was also estimated by assuming the thickness of the surface layer is the length of a typical Ce-O bond which is estimated as $0.2094 \mathrm{~nm} .{ }^{5}$ The volume of the surface $\left(V_{\mathrm{s}}\right)$ was then calculated for two Ce-O bond lengths to consider both surface and near surface sites:

$$
V_{\mathrm{s}}=4 / 3 \Pi\left(r^{2}-(r-(2 \times 0.2094))^{2}\right)
$$

From here $N_{\text {surf }}$ is easily calculated via a method analogous to equation S2:

$$
N_{\mathrm{NP}}=4 *\left(V_{\mathrm{s}} / V_{\mathrm{uc}}\right)
$$


Table S1. Nanoparticle statistics for various sizes of cerium oxide nanoparticles

\begin{tabular}{llllll}
\hline & & \multicolumn{2}{c}{ Method 1 $^{b}$} & \multicolumn{2}{c}{ Method 2 $^{c}$} \\
Diameter (nm) & $N_{\mathrm{NP}}{ }^{a}$ & $N_{\text {surf }}$ & $N_{\text {surf }} / N_{\mathrm{NP}}$ & $N_{\text {surf }}$ & $N_{\text {surf }} / N_{\mathrm{NP}}$ \\
\hline 1.6 & 54 & 52 & 0.95 & 48 & 0.89 \\
1.7 & 65 & 58 & 0.90 & 56 & 0.87 \\
1.8 & 77 & 65 & 0.85 & 65 & 0.85 \\
1.9 & 91 & 73 & 0.80 & 75 & 0.83 \\
2.0 & 106 & 81 & 0.76 & 85 & 0.80 \\
2.1 & 122 & 89 & 0.73 & 96 & 0.78 \\
2.2 & 141 & 97 & 0.69 & 107 & 0.76 \\
\hline 3.6 & 617 & 261 & 0.42 & 338 & 0.55 \\
4.0 & 846 & 322 & 0.38 & 428 & 0.51 \\
4.4 & 1126 & 390 & 0.35 & 528 & 0.47
\end{tabular}

${ }_{a}$ The total number of cerium atoms is estimated by approximating the nanoparticle as a sphere. ${ }^{b}$ The number of surface sites is estimated by approximating the surface as a symmetric truncated octahedron with (111) and (100) facets exposed. ${ }^{c}$ The number of surface sites is estimated by approximating the surface as a spherical shell with a thickness of two Ce-O bond lengths.

Applying the values above to OLE-Ce colloids is complicated by the distribution of nanoparticle sizes considered observed by TEM (see Figure S2). However, even after considering this range and two different methods for calculating the number of surface sites the percentage of surface cerium atoms changes significantly from Ce-1 and Ce-2 (69-95\%) to the larger Ce-L (35-55\%).

\subsection{ICP-MS Determinations of the Concentration of Cerium Atoms in OLE-Ce Colloids}

The concentration of cerium atoms in OLE-Ce stock solutions was determined by taking an aliquot $(<0.1$ $\mathrm{mL}$ ) of the THF stock solution and calcining the residual organic matter. The resulting yellow solid was digested in a $1 \mathrm{~mL}$ mixture of $1: 1$ concentrated $\mathrm{H}_{2} \mathrm{SO}_{4}$ and 50 wt. $\% \mathrm{H}_{2} \mathrm{O}_{2}$ (aq). We caution the reader that this mixture is hazardous so it should always be freshly prepared and quickly disposed, the volume should be kept to a minimum, and all manipulations should be done in a fumehood. Four samples were prepared via serial dilutions of the digested nanoparticles and the Fluka Ce standard. ICP-MS analysis of the samples by the method of standard additions gave the cerium concentration of the initial OLE-Ce stock solution. All concentration measurements shown in Table $\mathrm{S} 2$ were done starting from the calcination. 
Table S2. Compilation of ICP-MS analyses for different batches of nanoceria colloids including repeats.

\begin{tabular}{lll}
\hline Sample $^{a}$ & Concentration of Ce Atoms (mM) & $\mathrm{R}^{2 b}$ \\
\hline Ce-1 & 130.1 & 0.999 \\
Ce-1 & 131.1 & 0.999 \\
Ce-2 & 80.4 & 0.999 \\
Ce-2 & 85.3 & 0.999 \\
Ce-L & 146.6 & 0.999 \\
\hline
\end{tabular}

${ }^{a}$ For each entry sample preparation began by calcining an aliquot of the referenced stock solution. ${ }^{b}$ Propagation of error associated with fit gives a standard deviation less than $0.1 \mathrm{mM}$. As a result, final values and errors were determined by averaging data from multiple runs when possible.

\section{4 ${ }^{1}$ H NMR Determination of the Concentration of Oleate/Oleic Acid Capping Ligands}

The concentration of oleate/oleic acid capping ligands was measured for the Ce-1 colloid by adding $5 \mathrm{uL}$ $37 \mathrm{wt} \% \mathrm{HCl}_{(\mathrm{aq})}$ to an NMR sample containing [Ce atoms] $=9.1 \mathrm{mM}$ and [TMB] $=4.7 \mathrm{mM}$ in THF/THF$\mathrm{d}_{8}$. After this procedure all bound ligands are protonated and can be observed as free oleic acid in the ${ }^{1} \mathrm{H}$ NMR spectrum. The total amount of oleate/oleic acid in the as-prepared Ce-1 colloid was then quantified vs the TMB standard as $139 \mathrm{mM}$. This corresponds to roughly a 1:1 ratio between oleate/oleic acid and cerium atoms.

\section{X-ray Absorption Spectroscopy Experimental Details}

XAS measurements were performed at the Advanced Photon Source on the bending-magnet beamline 9BM-B with electron energy of $7 \mathrm{GeV}$ and average current of $100 \mathrm{~mA}$. The radiation was monochromatized by a Si (111) double-crystal monochromator. Fluorescence data were collected at the Ce $\mathrm{L}_{\mathrm{III}}$-edge (5723 eV) using a Vortex-METM multi-element Silicon drift detector, manufactured by SII NanoTechnology USA, Inc. Energies were calibrated to a $\mathrm{Cr}(5989.02 \mathrm{eV})$ foil reference. Samples were prepared in Charles Supper Company $1.0 \mathrm{~mm}$ OD quartz capillaries (wall thickness of $0.01 \mathrm{~mm}$ ) that are $80 \mathrm{~mm}$ in length. Addition of liquid samples to these capillaries was accomplished in an $\mathrm{N}_{2}$ glovebox atmosphere before sealing with Apiezon M-grease. Samples were then removed from the glovebox and sealed with hot wax. This procedure proved robust for preventing degradation of even the most air-sensitive samples. Iron bis(trimethylsilyl)amide (a pyrophoric liquid) was stable over the course of weeks in these capillaries. Sealed capillaries were shipped to APS and loaded onto a metal support with double sided tape (Figure S5). An X-Y positional fluorescence scan was then used to locate each sample prior to data collection.

Spectra were normalized using standard procedures in Athena. ${ }^{6}$ Edge positions were determined by locating the maximum of the first peak in the first-derivative spectrum. The ratio of cerium oxidation states was determined using Athena by fitting the XANES region $(-6$ to $+22 \mathrm{eV})$ with an arctan edge step and five unit normalized Gaussian functions, and using equation S8. Peak widths were held constant throughout the fitting procedure, while peak positions were allowed to float within a reasonable range as described in 
Table S3. ${ }^{7-9}$

$$
\% \mathrm{Ce}^{3+}=\frac{C}{A_{1}+A_{2}+B+C} \times 100 \%
$$


Table S3. Ce $\mathrm{L}_{\mathrm{III}}$-edge XANES assignments and information about peaks fits.

\begin{tabular}{lllllll}
\hline Peak & Assignment & Transition $^{a}$ & Center $(\mathrm{eV})$ & Function & Step & Width (eV) \\
\hline $\mathrm{A}_{1}$ & $\mathrm{Ce}^{4+}$ & $2 \mathrm{p}_{3 / 2} 4 \mathrm{f}^{0} \rightarrow 2 \mathrm{p}_{3 / 2} 4 \mathrm{f}^{0} 5 \mathrm{~d}^{*}$ & 5741.5 & Gaussian & -- & 2.0 \\
$\mathrm{~A}_{2}$ & $\mathrm{Ce}^{4+}$ & $2 \mathrm{p}_{3 / 2} 4 \mathrm{f}^{0} \rightarrow 2 \mathrm{p}_{3 / 2} 4 \mathrm{f}^{0} 5 \mathrm{~d}^{*}$ & 5737.7 & Gaussian & -- & 2.4 \\
$\mathrm{~B}$ & $\mathrm{Ce}^{4+}$ & $2 \mathrm{p}_{3 / 2} 4 \mathrm{f}^{1} \underline{\mathrm{L}} \rightarrow 2 \mathrm{p}_{3 / 2} 4 \mathrm{f}^{1} 5 \mathrm{~d}^{*} \underline{\underline{L}}$ & 5730.9 & Gaussian & -- & 2.6 \\
$\mathrm{C}$ & $\mathrm{Ce}^{3+}$ & $2 \mathrm{p}_{3 / 2} 4 \mathrm{f}^{0} \rightarrow 2 \mathrm{p}_{3 / 2} 4 \mathrm{f}^{1} 5 \mathrm{~d}^{*}$ & 5726.3 & Gaussian & -- & 2.2 \\
$\mathrm{D}$ & -- & $2 \mathrm{p}_{3 / 2} 4 \mathrm{f}^{0} \rightarrow 2 \mathrm{p}_{3 / 2} 4 \mathrm{f}^{*}$ & 5720.4 & Gaussian & -- & 2.0 \\
-- & -- & -- & 5725.0 & ArcTan & 1.0 & 0.94
\end{tabular}

${ }^{a}$ These transition assignments are based on previous work. ${ }^{7-9}$ An underlined state indicates a hole, whereas an asterisk indicates an excited electron.

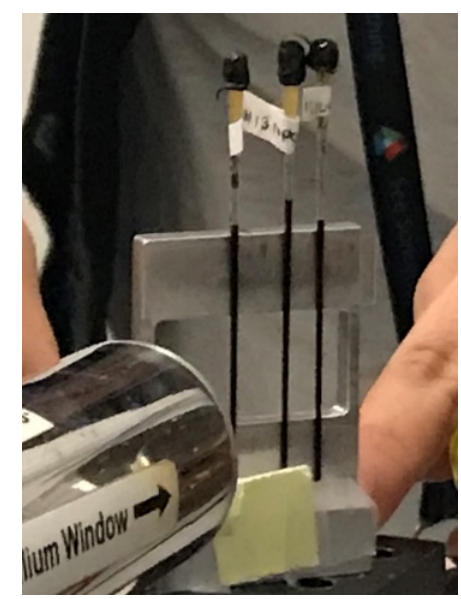

Figure S5. Set-up for XANES measurements of air-free solutions in quartz capillaries.

\section{Reversibility of OLE-Ce Redox Reactions}

\subsection{Limits of OLE-Ce Redox}

Addition of excess $\mathrm{H}_{2}$ DCAQ results in new peaks in the ${ }^{1} \mathrm{H}$ NMR below 0 ppm (Figure S6) and a loss of mass balance with respect to the total amount of $\mathrm{H}_{2} \mathrm{DCAQ}$ and DCAQ in solution from $>90 \%$ to $<80 \%$. As a result, the maximum extent of OLE-Ce reduction was defined by the maximum $\% \mathrm{Ce}^{3+}$ achieved via the oxidation of $\mathrm{H}_{2}$ DCAQ before new peaks below 0 ppm appeared in the ${ }^{1} \mathrm{H}$ NMR spectrum. These peaks likely correspond to a solution-phase oleate-bound cerium species based on the identification of similar peaks below zero for cerium(III) oleate (Figure S6).

The lowest $\% \mathrm{Ce}^{3+}$ characterized by both ${ }^{1} \mathrm{H}$ NMR and XANES for OLE-Ce colloids was achieved using excess DPPH as an oxidant and following production of DPPH-H which plateaus after several days. Both the minimum and maximum $\% \mathrm{Ce}^{3+}$ states were characterized by XANES and ${ }^{1} \mathrm{H}$ NMR (Table S4).

Additionally, OLE-Ce treated initially with excess meta-chloroperoxybenzoic acid ( $m$ CPBA) was observed to react subsequently with DPPH-H. Characterization of the reaction between OLE-Ce and $m$ CPBA was challenging as addition of excess $m$ CPBA to a solution of OLE-Ce causes displacement of oleate ligands such that significantly more free oleic acid is observed by ${ }^{1} \mathrm{H}$ NMR. We hypothesize that the excess $m \mathrm{CPBA}$ 
is binding to the nanoparticle surface as even upon addition of significant excesses of $m$ CPBA only the product meta-chlorobenzoic acid is observed by ${ }^{1} \mathrm{H}$ NMR. Additionally, $m$ CPBA decomposes in THF solutions by an unknown process which is accelerated in the presence of OLE-Ce. As a result, the extent of OLE-Ce oxidation by $m$ CPBA was characterized by following the decrease in DPPH-H ${ }^{1} \mathrm{H}$ NMR signal over time, which reaches a plateau. Loss of DPPH-H integration occurred concurrently with a change in solution color from orange (DPPH-H) to purple (DPPH). It is notable that $m \mathrm{CPBA}$ does not oxidize DPPH$\mathrm{H}$ in the absence of OLE-Ce. These data indicate that an equilibrium state is reached between OLE-Ce and the DPPH/DPPH-H $1 e^{-} / 1 \mathrm{H}^{+}$redox couple.

Table S4. Limits of OLE-Ce Redox by ${ }^{1} \mathrm{H}$ NMR

\begin{tabular}{llcc}
\hline Reactant $^{a}$ & Anchor $^{b}$ & $\Delta \% \mathrm{Ce}^{3+}$ & ${ }^{3} \mathrm{Ce}^{3+}$ by XANES \\
\hline $\mathrm{H}_{2} \mathrm{DCAQ}^{c}$ & as-prepared Ce-1 & $50-54 \%$ & -- \\
DPPH & as-prepared Ce-1 & $-6 \%$ & 23 \\
$m$ CPBA/DPPH-H & Ce-1/mCPBA & $1 \%$ & 21 \\
$\mathrm{H}_{2} \mathrm{DCAQ}^{2}$ & -- & -- & 76 \\
$\mathrm{H}_{2} \mathrm{DCAQ}^{d}$ & -- & -- & 72 \\
DPPH $^{d}$ & Ce-2 & $-10.7 \%$ & 16 \\
$\mathrm{H}_{2} \mathrm{DCAQ}^{e}$ & -- & -- & 30 \\
DPPH $^{e}$ & Ce-L & $-2.4 \%$ & 11
\end{tabular}

${ }^{a}$ In all cases reagents were added in excess unless otherwise specified. If two reagents are listed, they were added one after another so that the first reaction had reached completion before the second reagent was added. ${ }^{b}$ This refers to the state from which $\Delta \% \mathrm{Ce}^{3+}$ was calculated in ${ }^{1} \mathrm{H}$ NMR experiments. ${ }^{c}$ Addition was stoichiometric such that Ce1 was reduced to the maximum extent before new peaks appeared below $0 \mathrm{ppm}$ in the ${ }^{1} \mathrm{H}$ NMR spectrum (see Figure S6). ${ }^{d}$ Reacted with as-prepared Ce-2. ${ }^{e}$ Reacted with as-prepared Ce-L. 


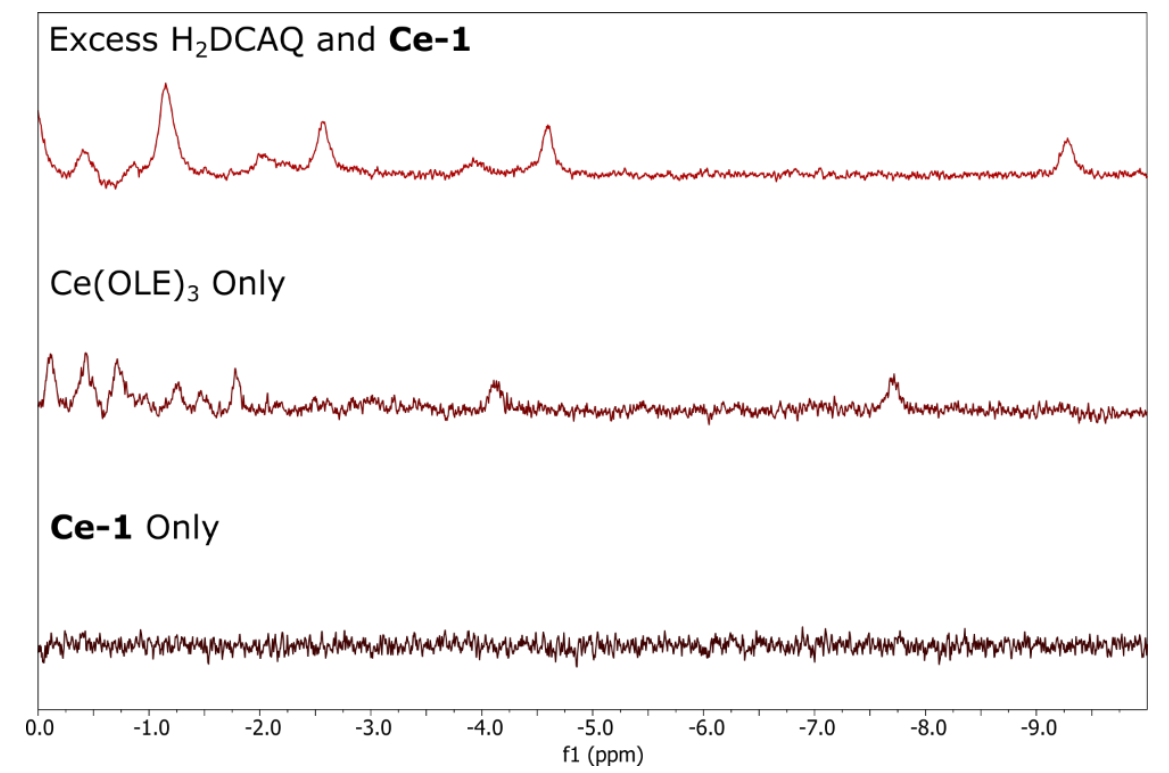

Figure S6. ${ }^{1} \mathrm{H}$ NMR samples of $\mathbf{C e}-\mathbf{1}$ (bottom), $\mathrm{Ce}(\mathrm{OLE})_{3}$ (middle), and $\mathbf{C e - 1}$ with excess $\mathrm{H}_{2} \mathrm{DCAQ}$ (top) in $\mathrm{d}_{8}$-THF. Ce-1/ $\mathrm{H}_{2}$ DCAQ sample contained $8 \mathrm{mM} \mathrm{Ce}$ atoms and $5 \mathrm{mM} \mathrm{H}_{2} \mathrm{DCAQ}$ before reaction and was measured one day after $\mathrm{H}_{2} \mathrm{DCAQ}$ addition.

\subsection{Linear Correlations between Molar Absorptivity and \%Ce $\mathrm{C}^{3+}$ for OLE-Ce Colloids}

Previous work by our group established that linear correlations are observed between the molar optical density of nanoceria colloids at $340 \mathrm{~nm}\left(\right.$ Molar $\left.\mathrm{OD}_{340}\right)$ and their $\% \mathrm{Ce}^{3+}$ determined by XANES. ${ }^{10}$ Here we build upon these studies by demonstrating linear correlations between Molar $\mathrm{OD}_{340}$ and $\% \mathrm{Ce}^{3+}$ determined by ${ }^{1} \mathrm{H}$ NMR for both Ce-1 and Ce-L (Figure 7).

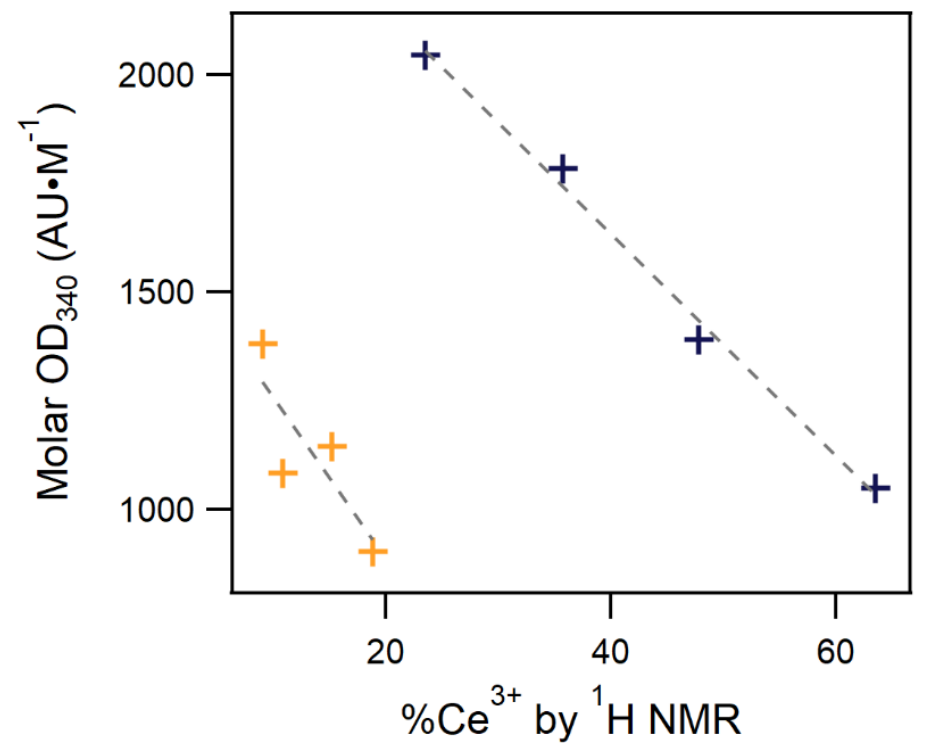

Figure 7. The $\% \mathrm{Ce}^{3+}$ of Ce-1 (blue) and Ce-L (orange) were varied using $\mathrm{H}_{2} \mathrm{DCAQ}$. Molar $\mathrm{OD}_{340}$ values were determined by subtracting contributions of leftover DCAQ (quantity determined by ${ }^{1} \mathrm{H}$ NMR) from the overall UV-Vis spectrum. 


\subsection{Redox Effects on OLE-Ce Size and Capping Ligands}

The effect of $\% \mathrm{Ce}^{3+}$ changes on OLE-Ce size were evaluated by DLS. Samples of Ce-2 were measured before and after photolysis and showed no measurable change in size distribution (Figure S8). See our previous work with OLE-Ce colloids for further information on photolysis procedures and evidence by TEM that redox state changes do not affect size. ${ }^{10}$

Additionally, the effect of $\% \mathrm{Ce}^{3+}$ on the oleate/oleic acid capping ligands was examined by following changes to their alkenyl C-H protons by ${ }^{1} \mathrm{H}$ NMR (Figure S9A). Results demonstrate that while changes to the peak do occur, they are reversible (Figure S9B). The origin of the loss oleate/oleic acid mass balance at high $\% \mathrm{Ce}^{3+}$ is unknown, but may be related to the increase in paramagnetic $\mathrm{Ce}^{3+}$ sites. In order to better explore this phenomenon spectra of Ce-1 were collected at $-50{ }^{\circ} \mathrm{C}$, where the contributions to the alkenyl $\mathrm{C}-\mathrm{H}$ peak deconvolute, while varying the $\% \mathrm{Ce}^{3+}$ in both directions (Figure $\mathrm{S} 10 \mathrm{~A}$ ). In these experiments only contributions from free oleic acid are visible at low $\% \mathrm{Ce}^{3+}$, but at high $\% \mathrm{Ce}^{3+}$ two new peaks steadily grow in while the quantity of oleic acid decreases (Figure S10B). While it is unclear what causes the growth of these new contributions to the spectrum, these changes are reversible upon oxidation of Ce-1.

Finally, the effect of redox changes on water ligands, and vice versa, was studied by ${ }^{1} \mathrm{H}$ NMR. A linear correlation between $\% \mathrm{Ce}^{3+}$ and $\left[\mathrm{H}_{2} \mathrm{O}\right]$ is observed (Figure $\mathrm{S} 11$ ). However, addition of $3 \AA$ sieves to remove $\mathrm{H}_{2} \mathrm{O}$ had no effect on the reversibility of redox reactions at Ce-1 (Figure S12). In summary, while there are clearly changes to the ligand sphere of OLE-Ce with changes in $\% \mathrm{Ce}^{3+}$ the studies we have performed indicate that these changes are largely reversible and have little effect on redox reactivity of OLE-Ce (see also Table S7).

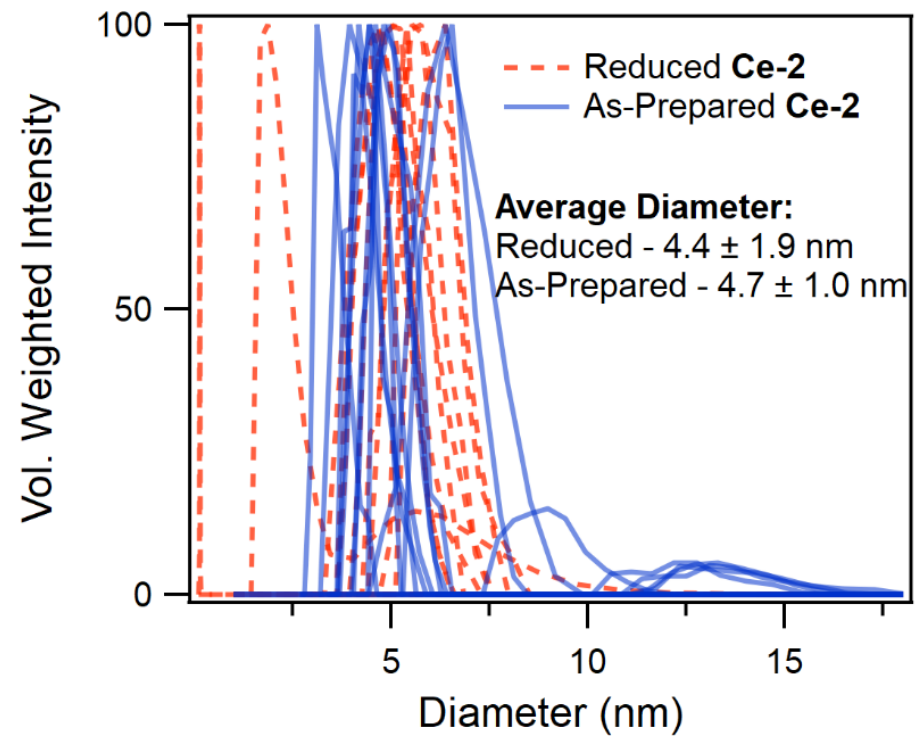

Figure S8. Sizing data of as-prepared (blue solid) and photochemically reduced (red dashed) Ce-2 by DLS. Each data set consists of 10 run (shown as separate peaks) that are plotted in terms of the diameter for the peak of Volume (Vol.) Weighted Intensity in each run. Calculation of the Vol. Weighted Intensity was achieved with Brookhaven Particle Solutions software. Samples were measured under air in glass cuvettes. 
A

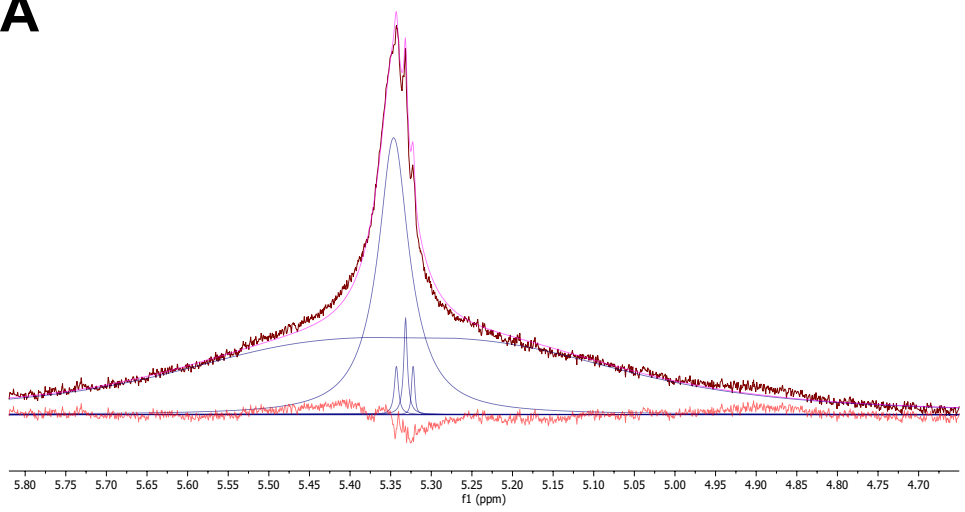

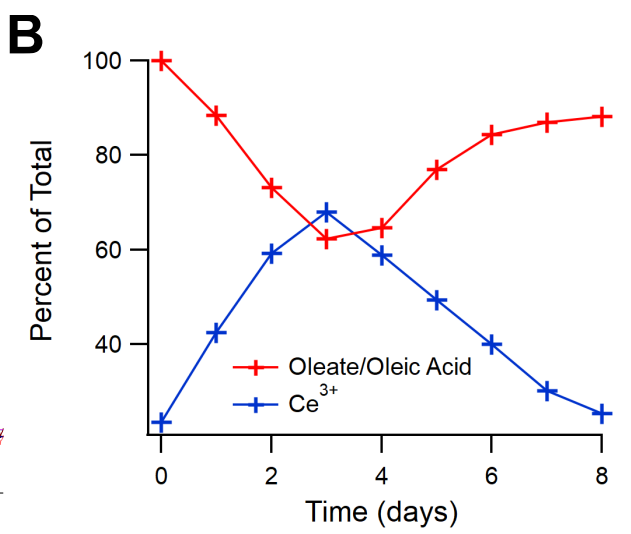

Figure S9. The sample solution contains as-prepared Ce-1 to which aliquots of $\mathrm{H}_{2} \mathrm{DCAQ}$ and DPPH stock solutions are added, respectively, to either increase or decrease $\% \mathrm{Ce}^{3+}$. (A) ${ }^{1} \mathrm{H} \mathrm{NMR}$ spectrum (dark red) of the alkenyl proton region of bound/free oleate/oleic acid fit to five voigt lineshapes (blue). The overall fit (pink) and residual (red) are also included. The two voigt lineshapes of significant integration are assigned to bound oleate/oleic acid, whereas the remaining three smaller lineshapes are assigned to free oleic acid based on spectra of just the free acid. Fits were done in MestReNova ${ }^{\circledR}$. (B) Trend between $\% \mathrm{Ce}^{3+}$ and changes in mass balance of alkenyl proton peaks assigned to bound oleate/oleic acid, as determined vs. an internal 1,3,5-trimethoxybenzene standard.
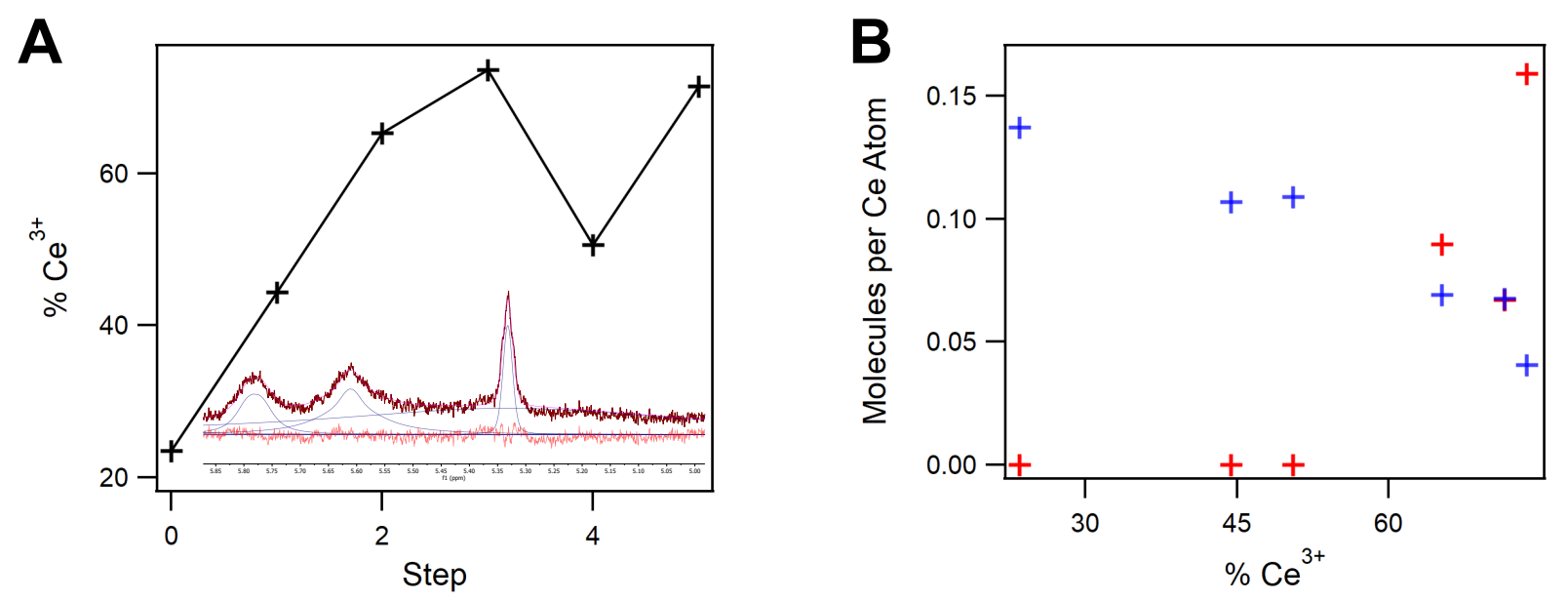

Figure S10. The sample solution contains as-prepared Ce-1 to which aliquots of $\mathrm{H}_{2} \mathrm{DCAQ}$ and DPPH stock solutions are added, respectively, to either increase or decrease $\% \mathrm{Ce}^{3+}$. All spectra were taken at $-50{ }^{\circ} \mathrm{C}$. In (A) the main plot describes how the $\% \mathrm{Ce}^{3+}$ of $\mathbf{C e}-\mathbf{1}$ was varied during this experiment. Each step refers to the addition of an aliquot of either $\mathrm{H}_{2}$ DCAQ or DPPH stock solutions in order to either increase or decrease $\% \mathrm{Ce}^{3+}$ in time. Additionally, the inset shows a representative ${ }^{1} \mathrm{H}$ NMR spectrum (dark red) of the alkenyl proton region of bound/free oleate/oleic acid for reduced Ce-1 fit to four voigt lineshapes (blue) where the first two peaks are unknown species (UL), which appear in roughly a 1:1 ratio, and the sharper feature to the right is assigned as free oleic acid (OA) based on spectra of just the pure acid. Fits were done in MestReNova $^{\circledR}$. (B) Plot of the amounts of UL (red) and OA (blue) per cerium atom, as determined vs. an internal 1,3,5-trimethoxybenzene standard, as a function of $\% \mathrm{Ce}^{3+}$. 


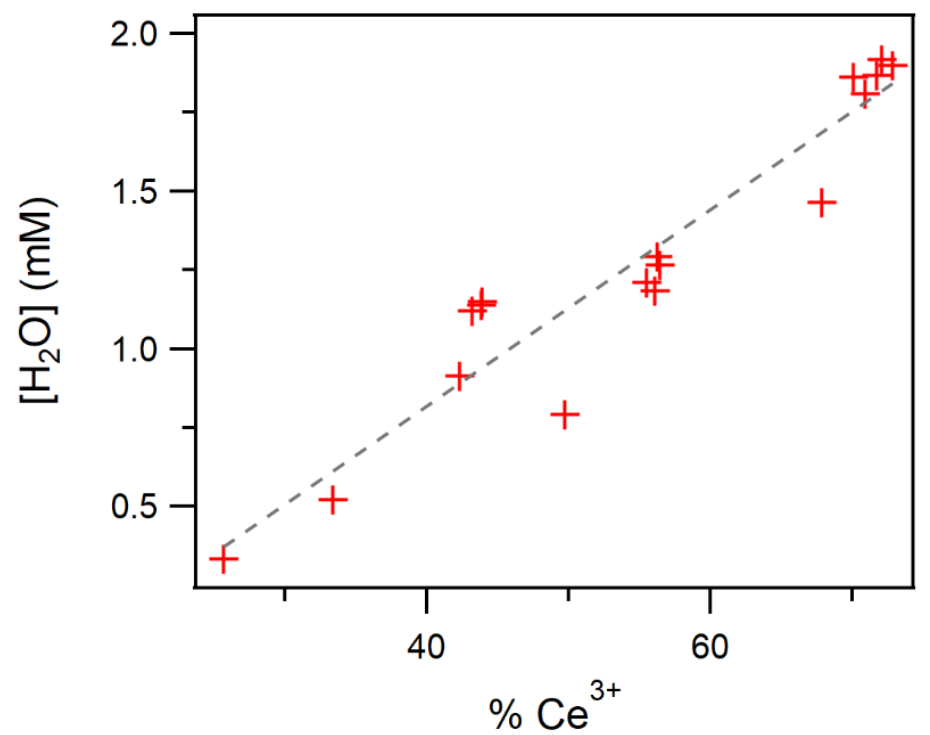

Figure S11. Data points are measured from ${ }^{1} \mathrm{H}$ NMR spectra of reactions between either $\mathrm{H}_{2} \mathrm{BQ}, \mathrm{H}_{2} \mathrm{DMQ}$, $\mathrm{H}_{2} \mathrm{NQ}$, or $\mathrm{H}_{2} \mathrm{DTNQ}(5 \mathrm{mM})$ and Ce-1 $([\mathrm{Ce}$ atoms $]=9 \mathrm{mM})$ in $\mathrm{d}_{8}-\mathrm{THF}$. The gray trace is a linear fit for reference. Changing the plot to units of $\left[\mathrm{H}_{2} \mathrm{O}\right]$ vs. $\left[\mathrm{Ce}^{3+}\right]$ gives a plot of slope 0.35 .

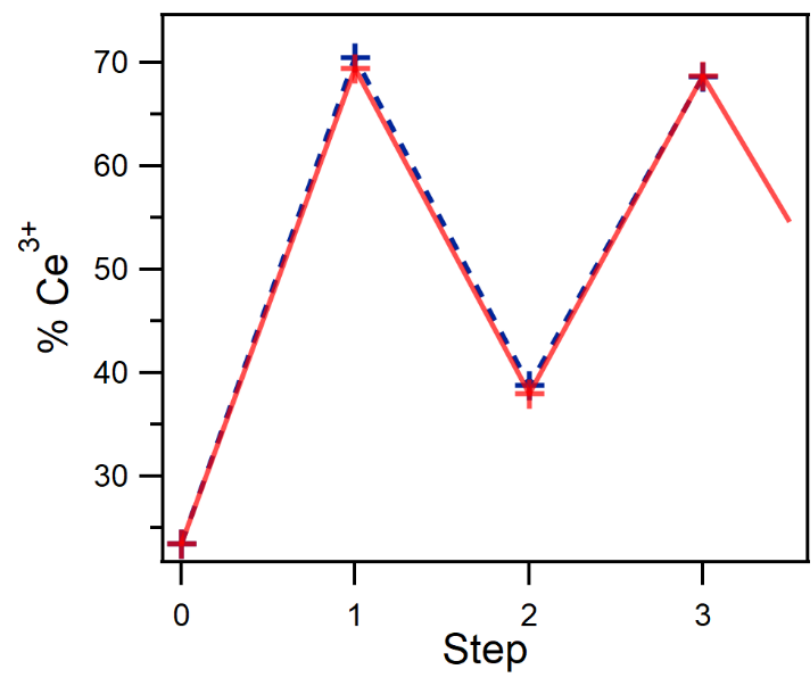

Figure S12. Redox cycling of Ce-1 using $\mathrm{H}_{2} \mathrm{DCAQ}$ as the reductant and DPPH as the oxidant. NMR samples were prepared using stock solutions of $\mathrm{H}_{2}$ DCAQ and DPPH in THF- $\mathrm{d}_{8}$. Trace of $\% \mathrm{Ce}^{3+}$ upon addition of set aliquots of $\mathrm{H}_{2} \mathrm{DCAQ}$ followed by DPPH in the presence (blue, dashed) and absence (red, solid) of $3 \AA$ sieves.

\section{Synthesis and Characterization of Organic PCET Reagents}

All PCET reagents except 2,7-di-tert-butyl-1,4-napthoquinone (DTNQ, Sigma Aldrich), 2,2-diphenyl-1picrylhydrazyl (DPPH, Sigma Aldrich), and 1,1-diphenyl-2-picrylhydrazine (DPPH-H, Sigma Aldrich) were purified before use. Quinones of a lower molecular weight (1,4-benzoquinone (BQ), 2,6-dimethyl1,4-benzoquinone (DMQ), 1,4-naphthoquinone (NQ)) were purified by sublimation. Additionally, 1,8- 
dichloro-9,10-anthraquinone was purified by recrystallization in a 1:1 mixture of $\mathrm{CHCl}_{3}$ and EtOH to give yellow needle-like crystals.

All hydroquinones except 1,4-hydroquinone were synthesized with few modifications from a reported literature procedure. ${ }^{11-14}$ All manipulations were performed under a glovebox $\mathrm{N}_{2}$ atmosphere in order to avoid oxidation of the hydroquinone product. Purified quinone $(\sim 0.3 \mathrm{mmol})$ dissolved in THF and excess sodium dithionite $\left(\mathrm{Na}_{2} \mathrm{~S}_{2} \mathrm{O}_{4}\right.$, technical grade (ca. 85\%), Fisher Scientific) $(\sim 3 \mathrm{mmol})$ dissolved in $\mathrm{H}_{2} \mathrm{O}$ were added to a $20 \mathrm{~mL}$ glass vial. The two-phase mixture was stirred vigorously for $>15$ minutes (color change noticeable within 1 minute). The mixture was then separated in a separatory funnel by adding $3 \mathrm{~mL}$ of $\mathrm{Et}_{2} \mathrm{O}$. The organic phase was then collected, and the aqueous phase was extracted with an additional $2 \times 3 \mathrm{~mL}$ $\mathrm{Et}_{2} \mathrm{O}$. The collected organics were then dried over $\mathrm{MgSO}_{4}$ and filtered. The leftover $\mathrm{MgSO}_{4}$ was also washed with $\sim 4 \mathrm{~mL} \mathrm{Et}_{2} \mathrm{O}$ to remove leftover hydroquinone. The compound was then isolated by removing volatile organics in vacuo and triturating with pentane. Most impurities in crude reaction mixtures could be removed with a pentane wash. Except for $\mathrm{H}_{2} \mathrm{DTNQ}$, all hydroquinones have been previously reported and the spectra reported here match previous reports.

\section{1,4-benzoquinone (BQ)}

${ }^{1} \mathrm{H}$ NMR $\left(400 \mathrm{MHz}, \mathrm{THF}-\mathrm{d}_{8}\right) \delta 6.76(\mathrm{~s}, 4 \mathrm{H})$

\section{1,4-hydroquinone $\left(\mathrm{H}_{2} \mathrm{BQ}\right)$}

${ }^{1} \mathrm{H}$ NMR (400 MHz, THF-d 8 ) $\delta 7.43(\mathrm{~s}, 2 \mathrm{H}), 6.52(\mathrm{~s}, 4 \mathrm{H})$

\section{2,6-dimethyl-1,4-benzoquinone (DMQ)}

${ }^{1} \mathrm{H}$ NMR (400 MHz, THF-d 8 ) $\delta 6.53(\mathrm{~s}, 2 \mathrm{H}), 1.99$ (s, 6H)

\section{2,6-dimethyl-1,4-hydroquinone ( $\left.\mathrm{H}_{2} \mathrm{DMQ}\right)$}

${ }^{1} \mathrm{H}$ NMR (400 MHz, THF-d $) \delta 7.23$ (s, 1H), 6.43 (s, 1H), 6.29 (s, 2H), 2.10 (s, 6H)

\section{1,4-naphthoquinone (NQ)}

${ }^{1} \mathrm{H}$ NMR (400 MHz, THF-d $) \delta 8.03(\mathrm{~m}, 2 \mathrm{H}), 7.78(\mathrm{~m}, 2 \mathrm{H}), 6.98(\mathrm{~s}, 2 \mathrm{H})$

\section{1,4-dihydroxynapthalene $\left(\mathrm{H}_{2} \mathrm{NQ}\right)$}

${ }^{1} \mathrm{H}$ NMR (400 MHz, THF-d 8 ) $\delta 8.10(\mathrm{~m}, 2 \mathrm{H}), 8.09$ (s, 2H, -OH), 7.34 (m, 2H), $6.53(\mathrm{~s}, 2 \mathrm{H})$

\section{2,7-di-tert-butyl-1,4-naphthoquinone (DTNQ)}

${ }^{1} \mathrm{H}$ NMR (400 MHz, THF-d 8 ) $\delta 8.12(\mathrm{~s}, 1 \mathrm{H}), 7.92(\mathrm{~d}, 1 \mathrm{H}), 7.81(\mathrm{~d}, 1 \mathrm{H}), 6.79(\mathrm{~s}, 1 \mathrm{H}), 1.39$ (s, 9H), 1.37 (s, 9H)

\section{2,7-di-tert-butyl-1,4-dihydroxynaphthalene ( $\left.\mathrm{H}_{2} \mathrm{DTNQ}\right)$}

${ }^{1} \mathrm{H}$ NMR (600 MHz, THF-d 8 ) $\delta 8.04(\mathrm{~s}, 1 \mathrm{H}), 8.01$ (d, 1H), 7.98 (d, 1H), $7.43(\mathrm{dd}, 1 \mathrm{H}), 7.17$ (s, 1H), 6.73 (s, 1H), $1.48(\mathrm{~s}, 9 \mathrm{H}), 1.42(\mathrm{~s}, 9 \mathrm{H})$

${ }^{13} \mathrm{C}$ NMR (150 MHz, THF-d 8 ) $\delta 148.12$ (s, 1C), 147.63 (s, 1C), 143.79 (s, 1C), 133.04 (s, 1C), 128.79 (s, 1C), 123.37 (s, 1C), 123.08 (s, 1C), 123.06 (s, 1C), 116.91 (s, 1C), 107.49 (s, 1C), 35.87 (s, 1C), 35.64 (s, 1C), $31.93(\mathrm{~s}, 9 \mathrm{C}), 30.77(\mathrm{~s}, 9 \mathrm{C})$

\section{1,8-dichloro-9,10-anthraquinone (DCAQ)}

${ }^{1} \mathrm{H}$ NMR (400 MHz, THF-d $) \delta 8.18(\mathrm{~d}, 2 \mathrm{H}), 7.85(2 \mathrm{H}, \mathrm{d}), 7.71(\mathrm{t}, 2 \mathrm{H})$ 


\section{1,8-dichloro-9,10-dihydroxyanthracene ( $\left.\mathrm{H}_{2} \mathrm{DCAQ}\right)$}

${ }^{1} \mathrm{H}$ NMR (400 MHz, THF-d 8 ) $\delta 8.59(\mathrm{~s}, 1 \mathrm{H}), 8.52(\mathrm{~s}, 1 \mathrm{H}), 8.30(\mathrm{~d}, 2 \mathrm{H}), 7.46(\mathrm{~d}, 2 \mathrm{H}), 7.28(\mathrm{t}, 2 \mathrm{H})$

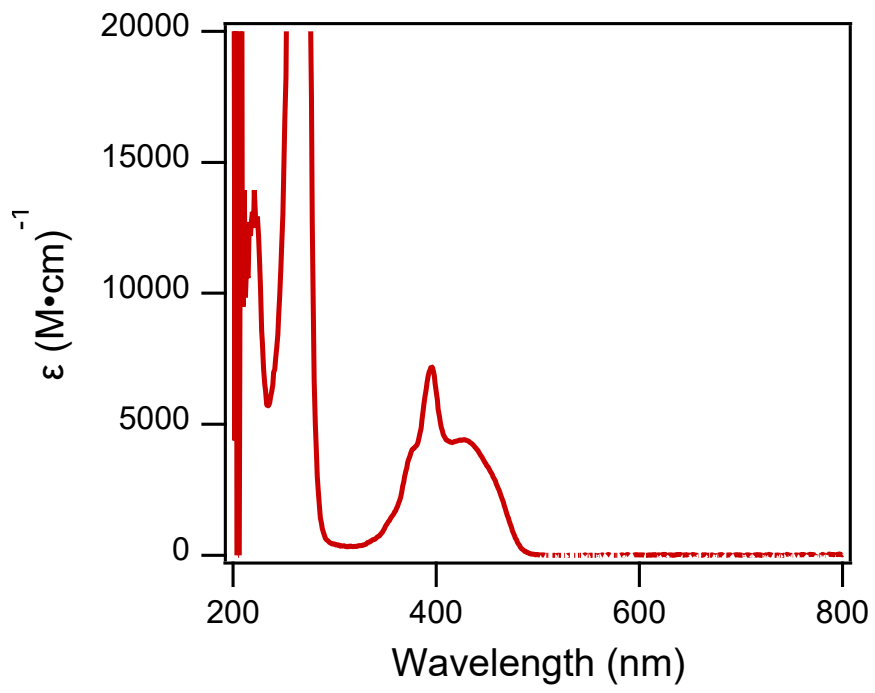

Figure S13. Normalized UV-Vis spectrum of $\mathrm{H}_{2} \mathrm{DCAQ}$ in THF. Characteristic absorbance feature at $\sim 400$ $\mathrm{nm}$ matches previous reports. ${ }^{14}$

\section{1,1-diphenyl-2-picrylhydrazine (DPPH-H)}

${ }^{1} \mathrm{H}$ NMR (400 MHz, THF-d 8 ) $\delta 10.55$ (s, 1H, -NH), 9.09 (s, 1H), 8.73 (s, 1H), 7.29 (t, 4H), 7.19 (d, 4H), $7.10(\mathrm{t}, 2 \mathrm{H})$

\section{Establishing Equilibrium between OLE-Ce and PCET Reagents in THF}

\section{1 ${ }^{1}$ H NMR spectra for Equilibrium of OLE-Ce and PCET Reductants}

These spectra are typical of the plateau region for equilibration experiments for the respective PCET reagents. Spectra below detail before addition of Ce-1 and at plateau region with Ce-1 ${ }^{1} \mathrm{H}$ NMR spectra for each equilibration. 


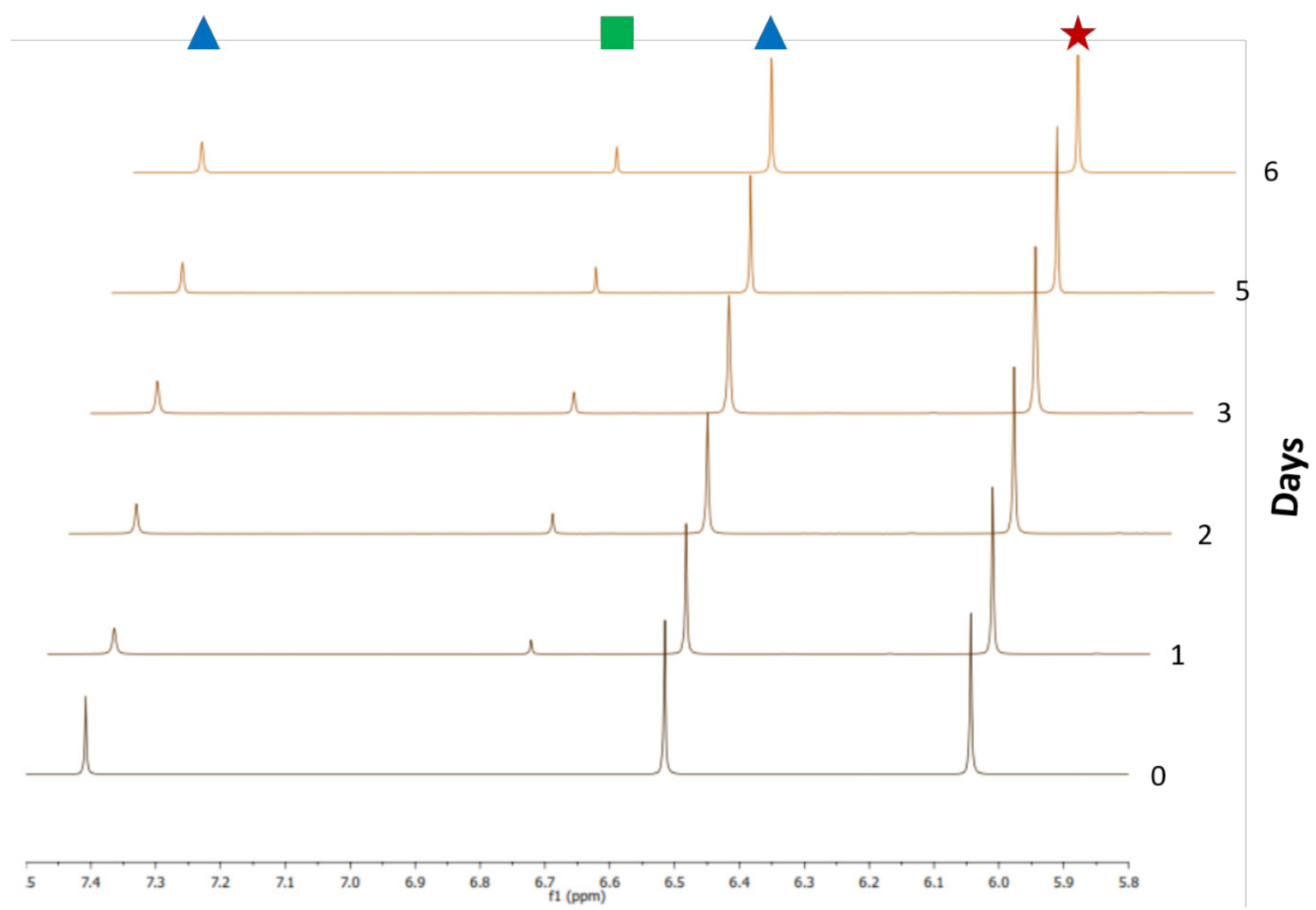

Figure S14. Time course of equilibration between $\mathbf{C e}-1$ and $\mathrm{H}_{2} \mathrm{BQ}$. Initial concentrations of the reaction mixture were $[\mathrm{Ce}$ atoms $]=9.0 \mathrm{mM},[\mathrm{TMB}]=8.2 \mathrm{mM}$, and $\left[\mathrm{H}_{2} \mathrm{BQ}\right]=5.5 \mathrm{mM}$. The TMB peak (red star), hydroquinone peak (blue triangle), and quinone peak (green square) chosen for integration are denoted. 


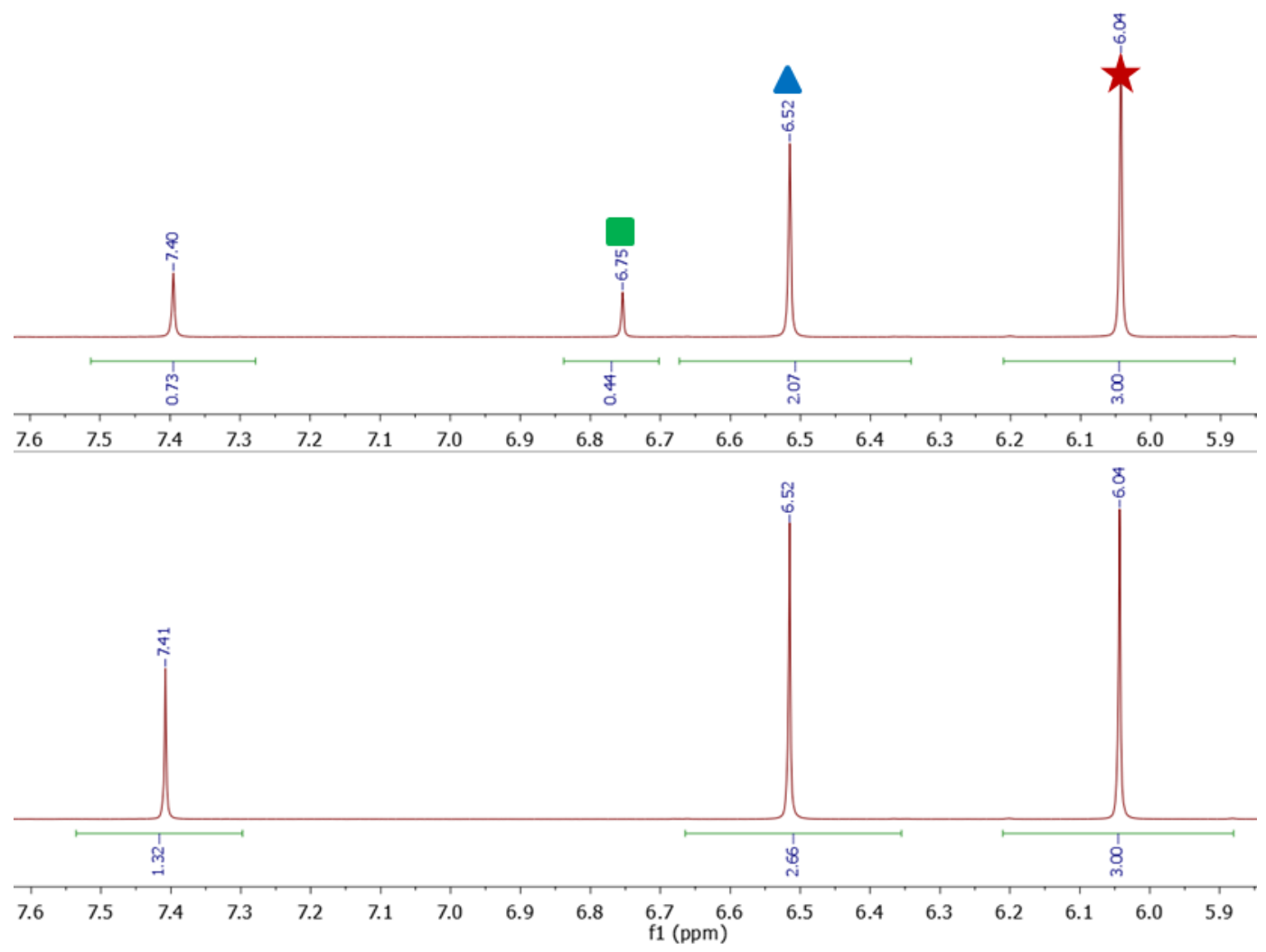

Figure S15. Initial (bottom) and final (top) ${ }^{1} \mathrm{H}$ NMR spectra of equilibration between Ce-1 and $\mathrm{H}_{2} \mathrm{BQ}$. Initial concentrations in the reaction mixture were $[\mathrm{Ce}$ atoms $]=9.0 \mathrm{mM},[\mathrm{TMB}]=8.2 \mathrm{mM}$, and $\left[\mathrm{H}_{2} \mathrm{BQ}\right]=$ $5.5 \mathrm{mM}$. The TMB peak (red star), hydroquinone peak (blue triangle), and quinone peak (green square) chosen for integration are denoted. 


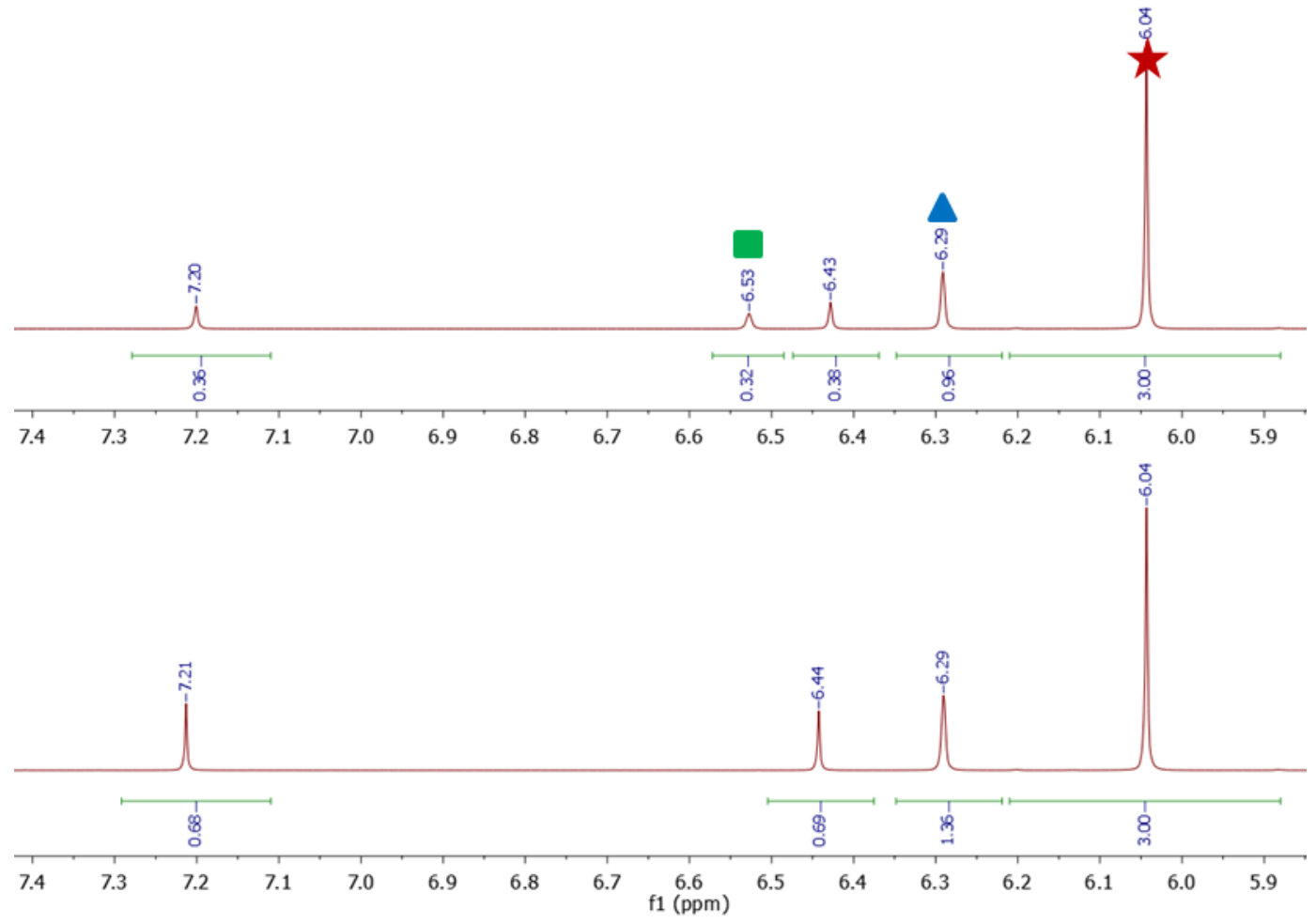

Figure S16. Initial (bottom) and final (top) ${ }^{1} \mathrm{H}$ NMR spectra of equilibration between Ce-1 and $\mathrm{H}_{2} \mathrm{DMQ}$. Initial concentrations in the reaction mixture were $\left[\mathrm{Ce}\right.$ atoms] $=9.0 \mathrm{mM},[\mathrm{TMB}]=8.2 \mathrm{mM}$, and $\left[\mathrm{H}_{2} \mathrm{DMQ}\right]$ $=5.6 \mathrm{mM}$. The TMB peak (red star), hydroquinone peak (blue triangle), and quinone peak (green square) chosen for integration are denoted. 


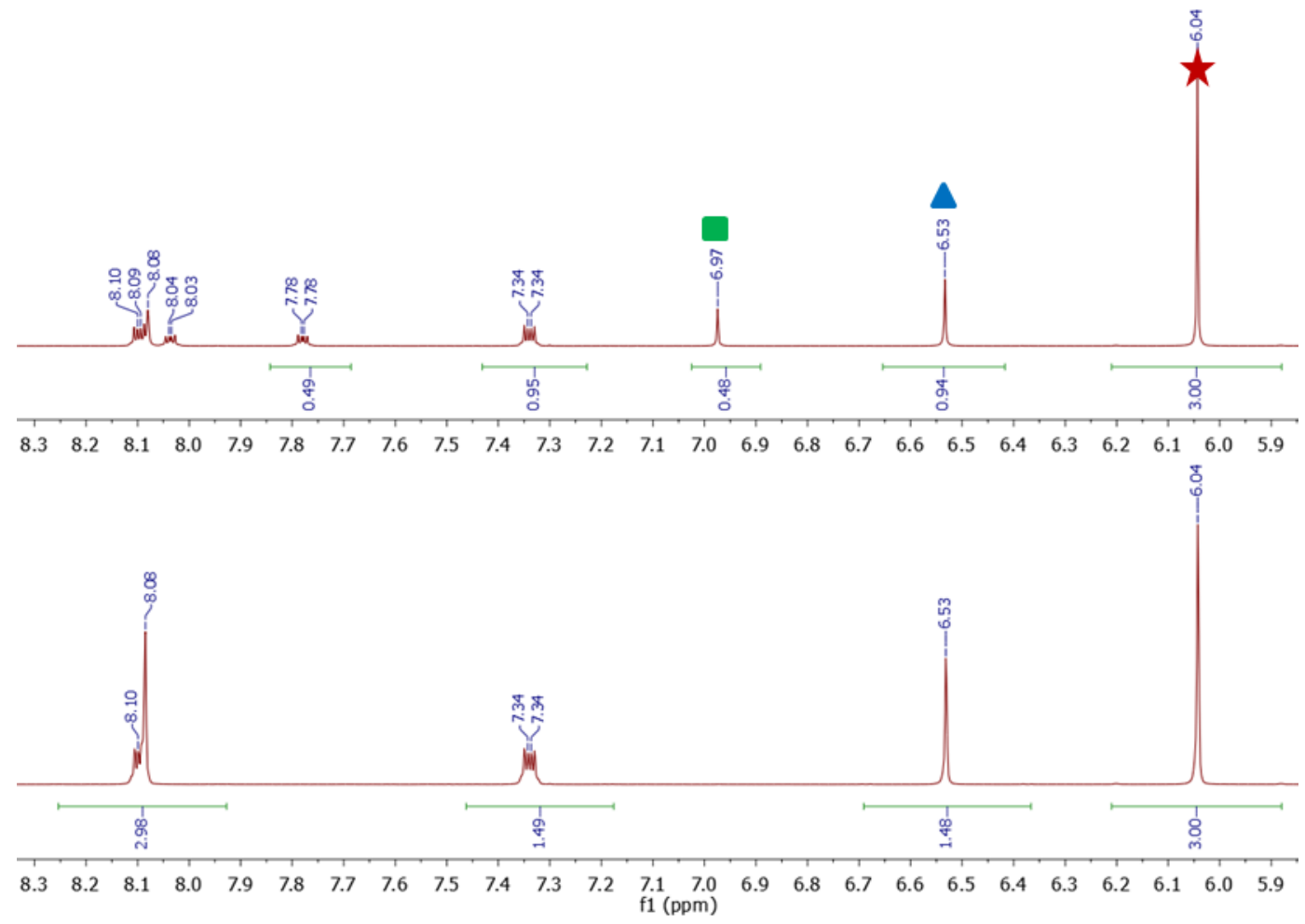

Figure S17. Initial (bottom) and final (top) ${ }^{1} \mathrm{H}$ NMR spectra of equilibration between Ce-1 and $\mathrm{H}_{2} \mathrm{NQ}$. Initial concentrations in the reaction mixture were $[\mathrm{Ce}$ atoms $]=9.0 \mathrm{mM},[\mathrm{TMB}]=7.2 \mathrm{mM}$, and $\left[\mathrm{H}_{2} \mathrm{NQ}\right]=$ $5.3 \mathrm{mM}$. The TMB peak (red star), hydroquinone peak (blue triangle), and quinone peak (green square) chosen for integration are denoted. 

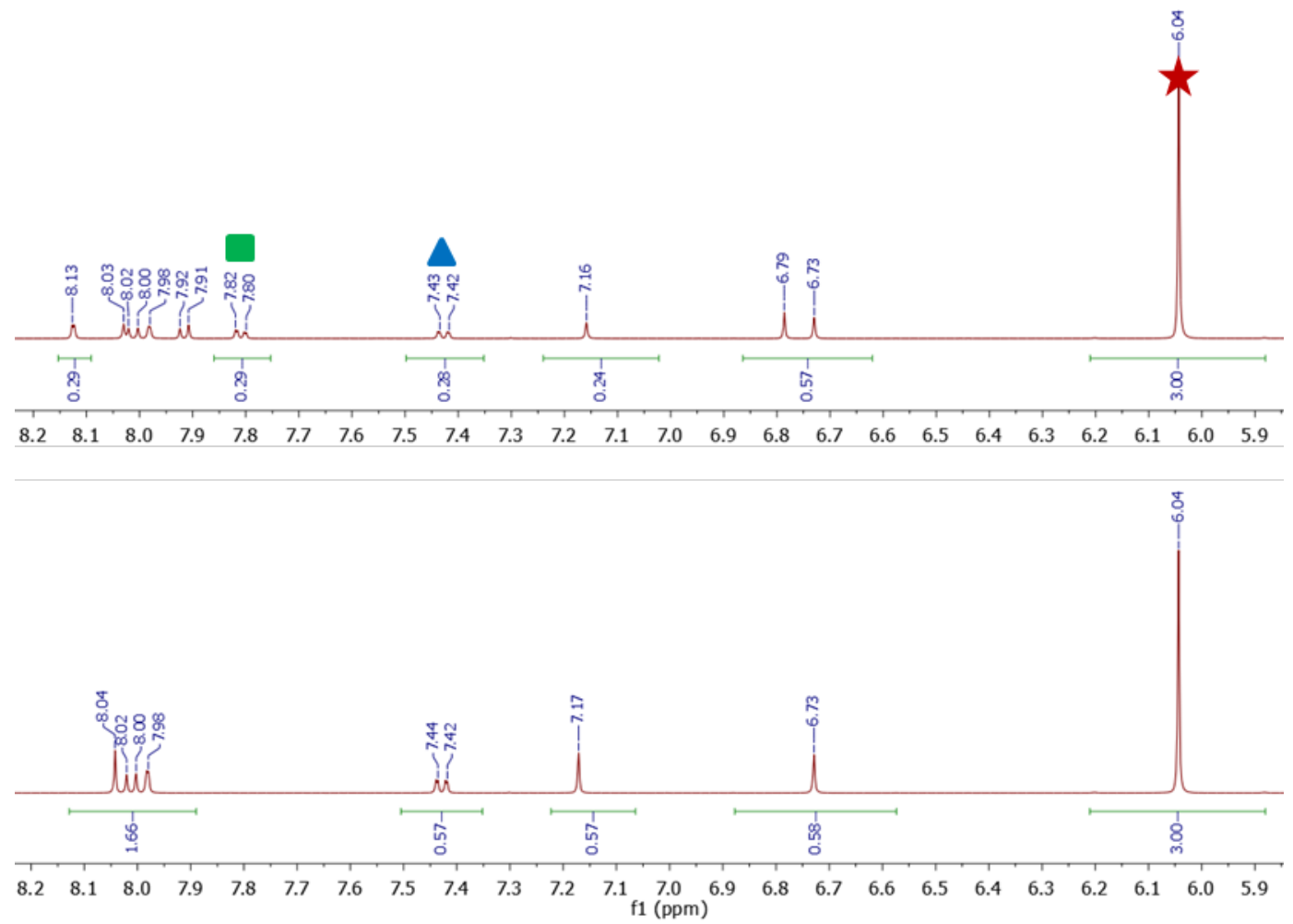

Figure S18. Initial (bottom) and final (top) ${ }^{1} \mathrm{H}$ NMR spectra of equilibration between Ce-1 and $\mathrm{H}_{2} \mathrm{DTNQ}$. Initial concentrations in the reaction mixture were $[\mathrm{Ce}$ atoms $]=9.0 \mathrm{mM}$, [TMB] $=7.6 \mathrm{mM}$, and $\left[\mathrm{H}_{2} \mathrm{DTNQ}\right]$ $=4.4 \mathrm{mM}$. The TMB peak (red star), hydroquinone peak (blue triangle), and quinone peak (green square) chosen for integration are denoted.

\section{$6.2{ }^{1} H$ NMR spectra for Equilibrium of OLE-Ce and PCET Oxidants}

These spectra are typical of the plateau region for equilibration experiments. For the respective quinones, spectra below detail the ${ }^{1} \mathrm{H}$ NMR spectra of OLE-Ce reduced by a stoichiometric amount of $\mathrm{H}_{2} \mathrm{DCAQ}$ before the addition of quinone and at plateau region with OLE-Ce after addition. 


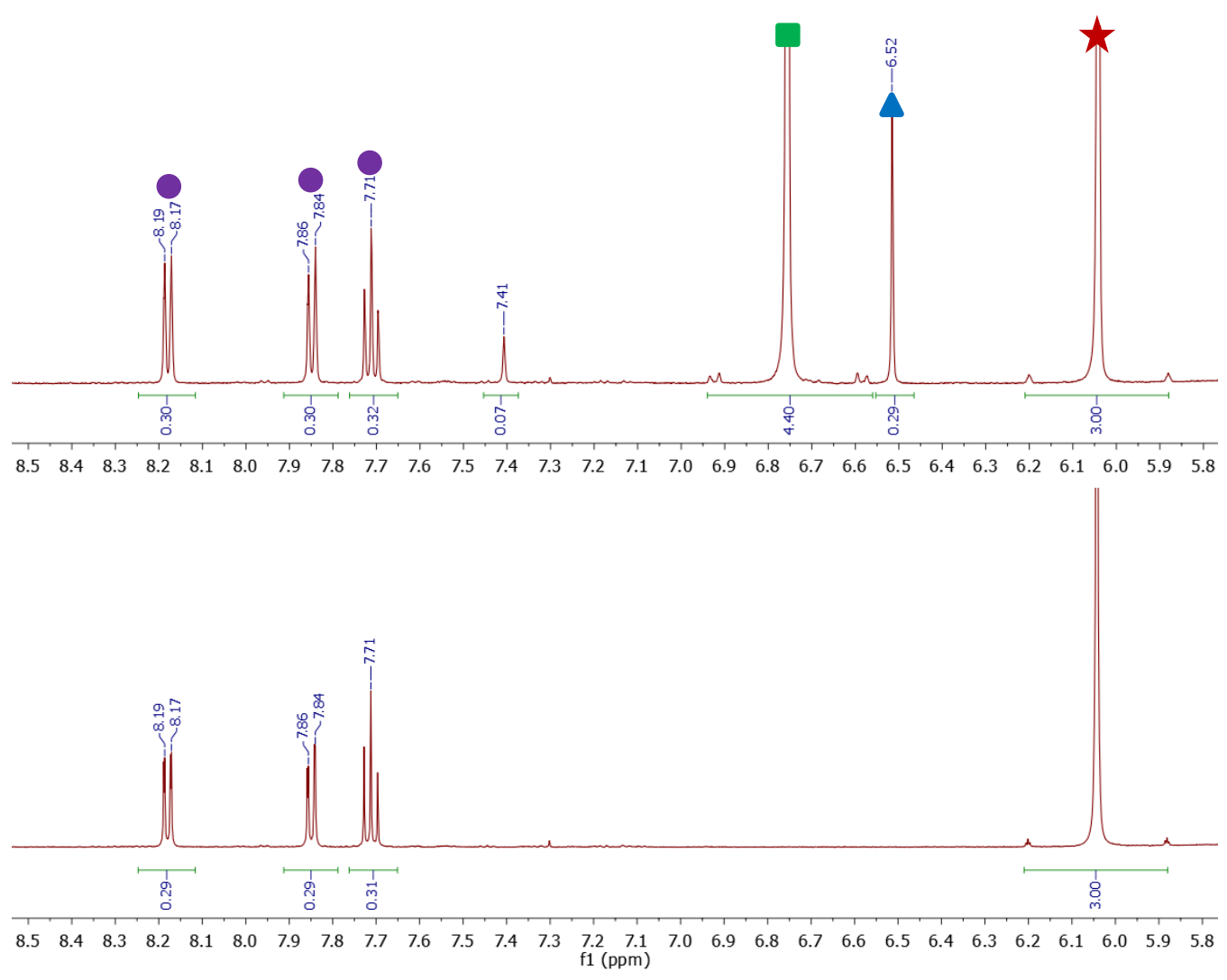

Figure S19. Initial (top) and final (bottom) ${ }^{1} \mathrm{H}$ NMR spectra of equilibration between reduced Ce-1 and $\mathrm{BQ}$. Initial concentrations in the reaction mixture after reduction of Ce-1 with $\mathrm{H}_{2} \mathrm{DCAQ}$ were [Ce atoms] $=9.1 \mathrm{mM},[\mathrm{TMB}]=7.9 \mathrm{mM},[\mathrm{DCAQ}]=1.1 \mathrm{mM}$, and $[\mathrm{BQ}]=9.3 \mathrm{mM}$. The TMB peak (red star), hydroquinone peak (blue triangle), quinone peak (green square), and DCAQ peaks (purple circle) chosen for integration are denoted. 


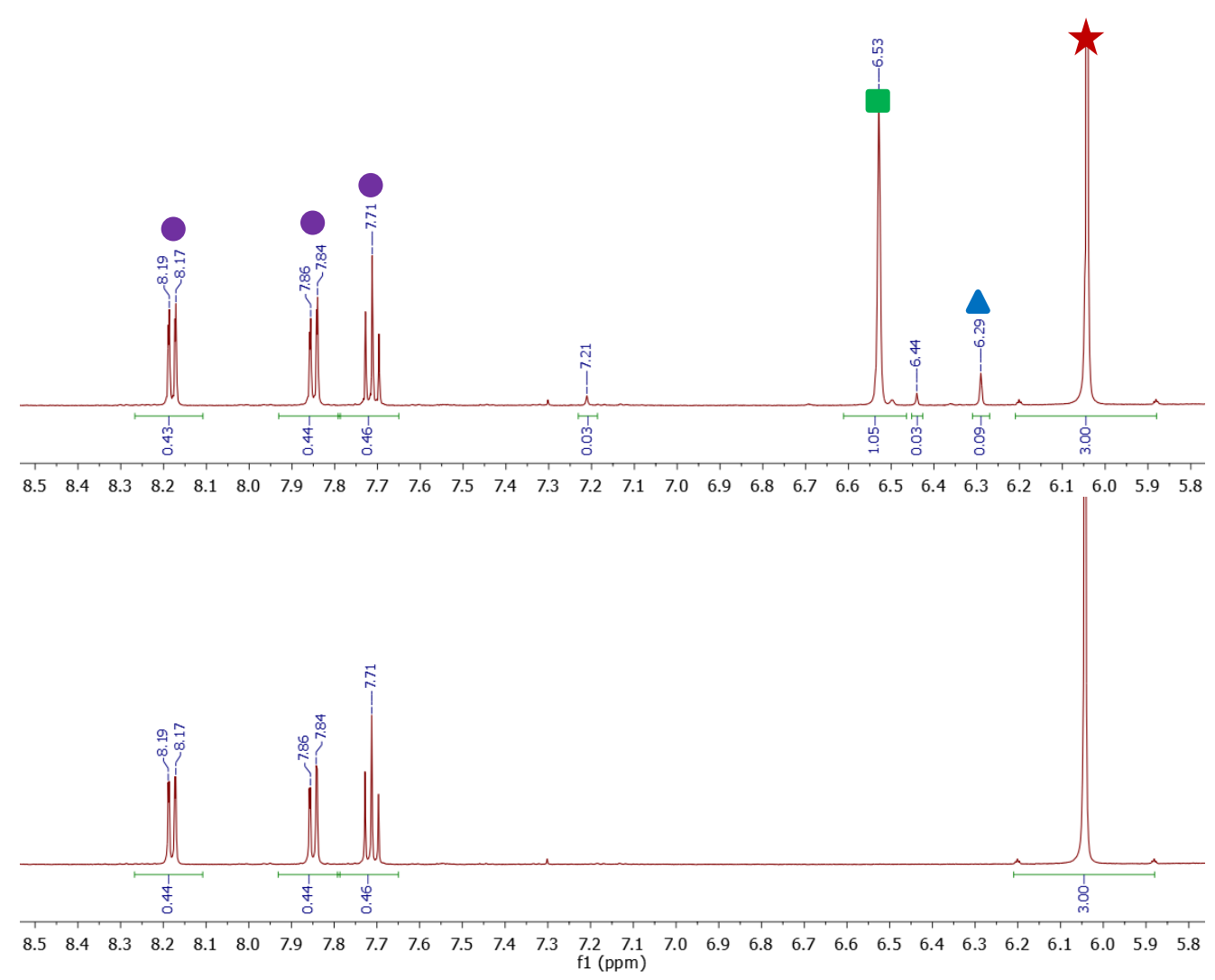

Figure S20. Initial (top) and final (bottom) ${ }^{1} \mathrm{H}$ NMR spectra of equilibration between reduced Ce-1 and DMQ. Initial concentrations in the reaction mixture after reduction of Ce-1 with $\mathrm{H}_{2}$ DCAQ were [Ce atoms] $=9.1 \mathrm{mM},[\mathrm{TMB}]=7.4 \mathrm{mM},[\mathrm{DCAQ}]=1.7 \mathrm{mM}$, and $[\mathrm{DMQ}]=4.3 \mathrm{mM}$. The TMB peak (red star), hydroquinone peak (blue triangle), quinone peak (green square), and DCAQ peaks (purple circle) chosen for integration are denoted. 

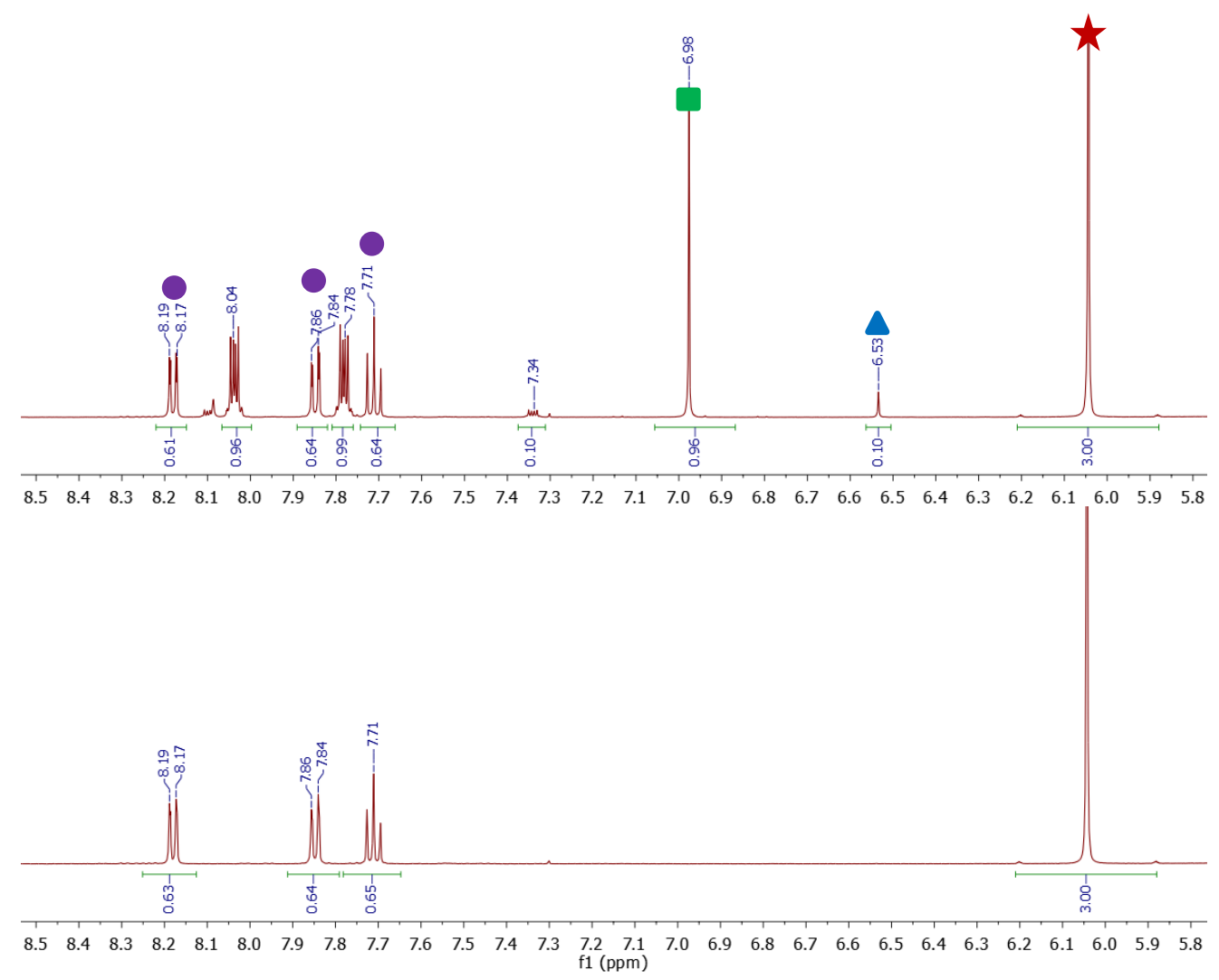

Figure S21. Initial (top) and final (bottom) ${ }^{1} \mathrm{H}$ NMR spectra of equilibration between reduced Ce-1 and NQ. Initial concentrations in the reaction mixture after reduction of Ce-1 with $\mathrm{H}_{2} \mathrm{DCAQ}$ were [Ce atoms] $=9.1 \mathrm{mM},[\mathrm{TMB}]=7.0 \mathrm{mM},[\mathrm{DCAQ}]=2.1 \mathrm{mM}$, and $[\mathrm{NQ}]=7.5 \mathrm{mM}$. The TMB peak (red star), hydroquinone peak (blue triangle), quinone peak (green square), and DCAQ peaks (purple circle) chosen for integration are denoted. 

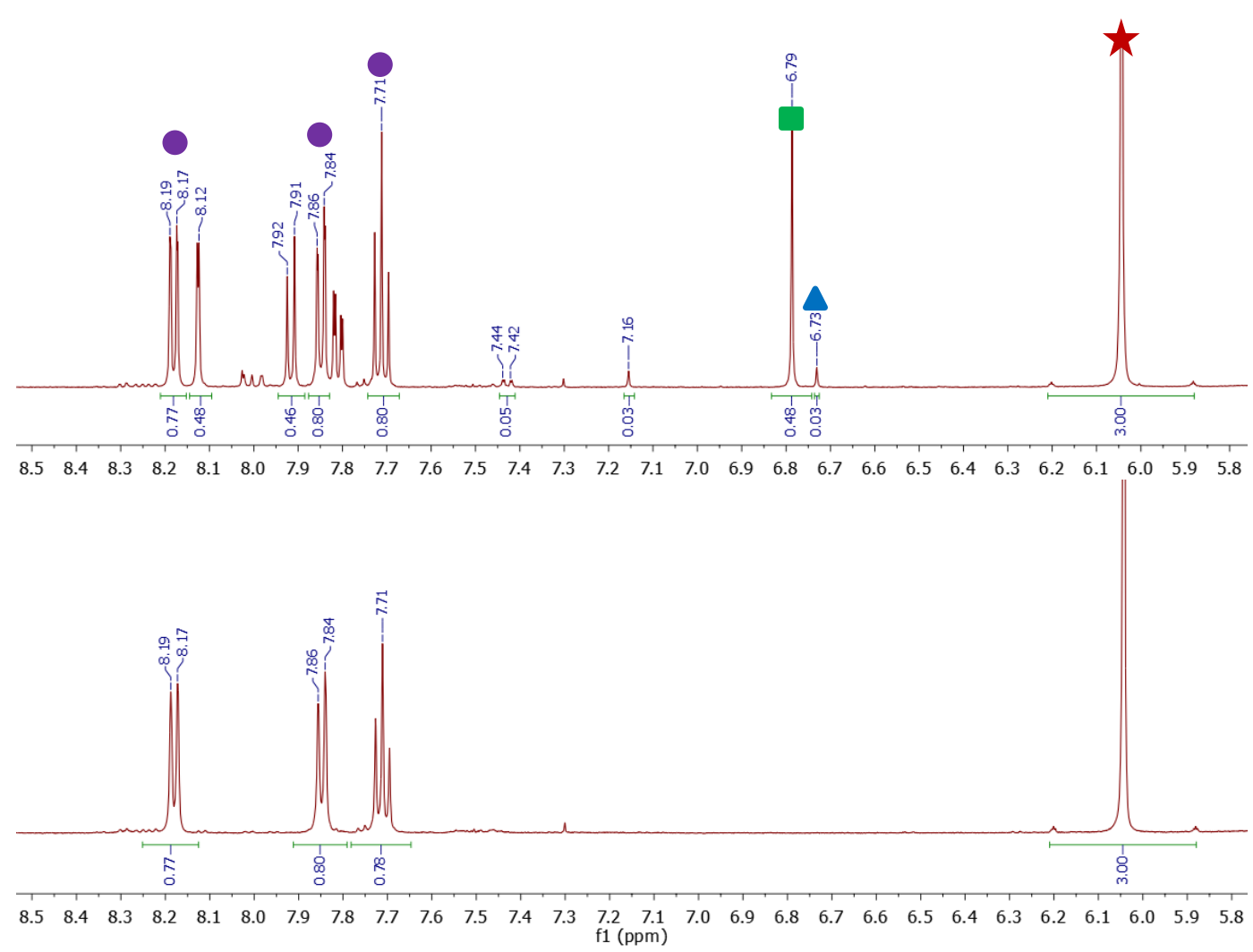

Figure S22. Initial (top) and final (bottom) ${ }^{1} \mathrm{H}$ NMR spectra of equilibration between reduced Ce-1 and DTNQ. Initial concentrations in the reaction mixture after reduction of Ce-1 with $\mathrm{H}_{2} \mathrm{DCAQ}$ were [Ce atoms $]=9.1 \mathrm{mM},[\mathrm{TMB}]=6.5 \mathrm{mM},[\mathrm{DCAQ}]=2.4 \mathrm{mM}$, and $[\mathrm{DTNQ}]=3.7 \mathrm{mM}$. The TMB peak (red star), hydroquinone peak (blue triangle), quinone peak (green square), and DCAQ peaks (purple circle) chosen for integration are denoted. 

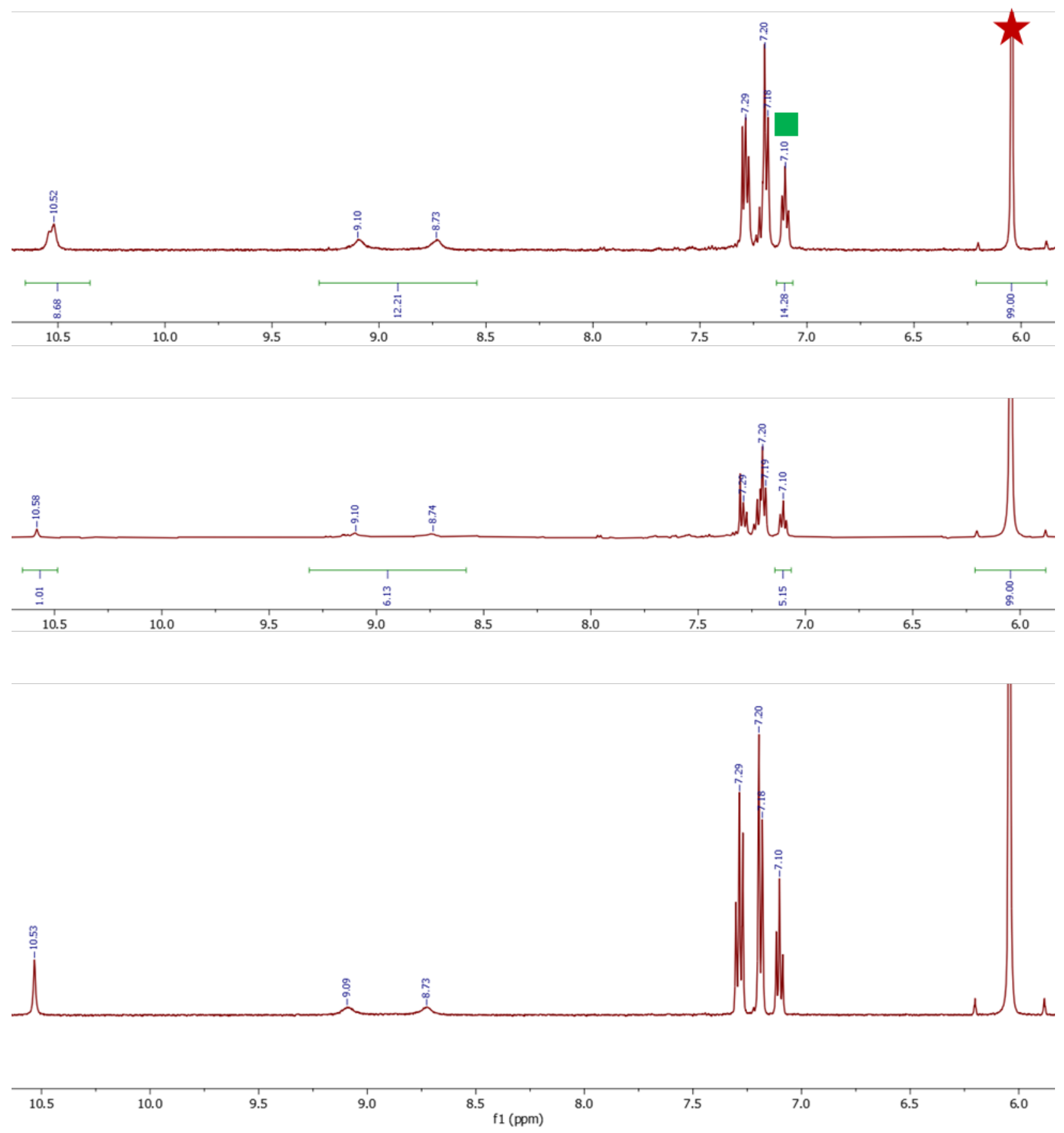

Figure 23. ${ }^{1} \mathrm{H}$ NMR spectra of DPPH-H (bottom), DPPH before addition of Ce-1 (middle), and after addition (top). Initial concentrations in the reaction mixture after addition of Ce-1 were [Ce atoms] $=11.5$ $\mathrm{mM},[\mathrm{TMB}]=4.6 \mathrm{mM},[\mathrm{DPPH}-\mathrm{H}]=0.4 \mathrm{mM}$, and $[\mathrm{DPPH}]=4.1 \mathrm{mM}$. The TMB peak (red star) and DPPH$\mathrm{H}$ peak chosen for integration are denoted. The initial [DPPH] was estimated based on the known weight of solid added and the assumption that all mass was from either DPPH or DPPH-H impurities.

\subsection{Compiled ${ }^{1} H$ NMR and XAS Data for Equilibrations}

Time courses of ${ }^{1} \mathrm{H}$ NMR data are compiled along with the associated fluctuations in organic reagent and product mass balance for reactions between OLE-Ce colloids and both quinones and hydroquinones (Figure 
S24). Compilations of XANES spectra of equilibrated OLE-Ce samples are also included (Figure S25). Finally, the values from all OLE-Ce equilibrations are compiled for both ${ }^{1} \mathrm{H}$ NMR (Table S5) and XANES studies (Table S6).

A
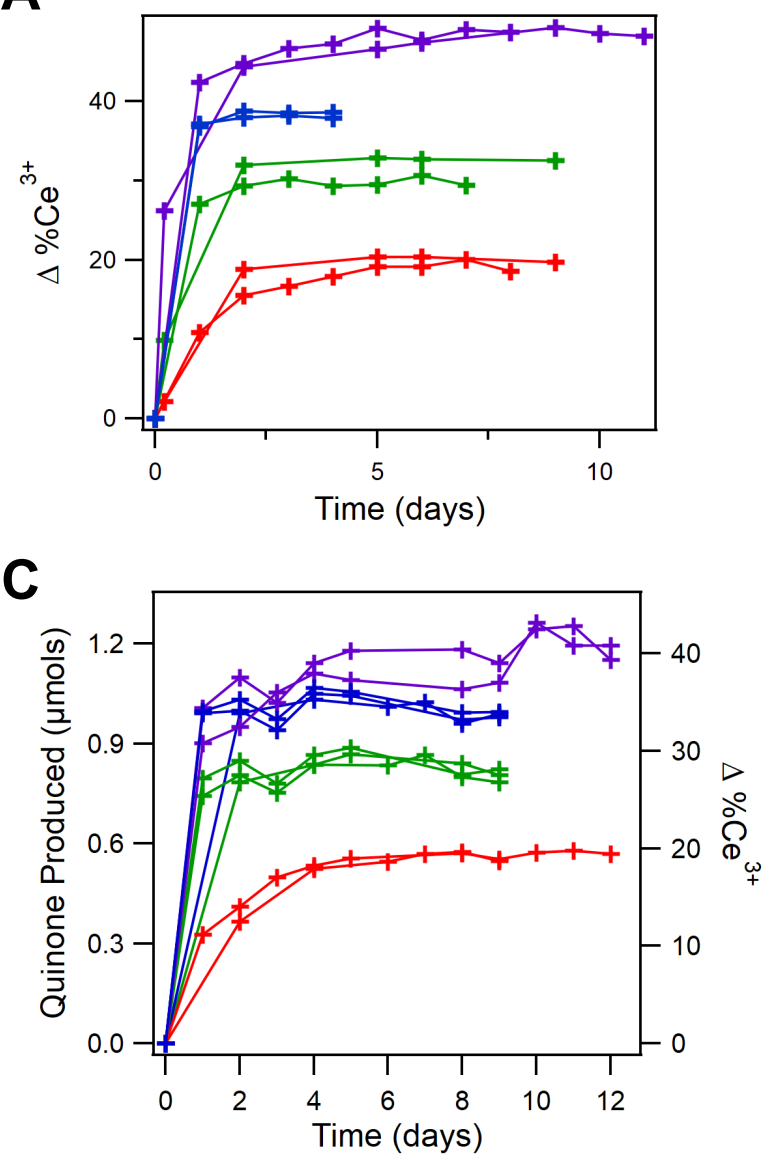

B

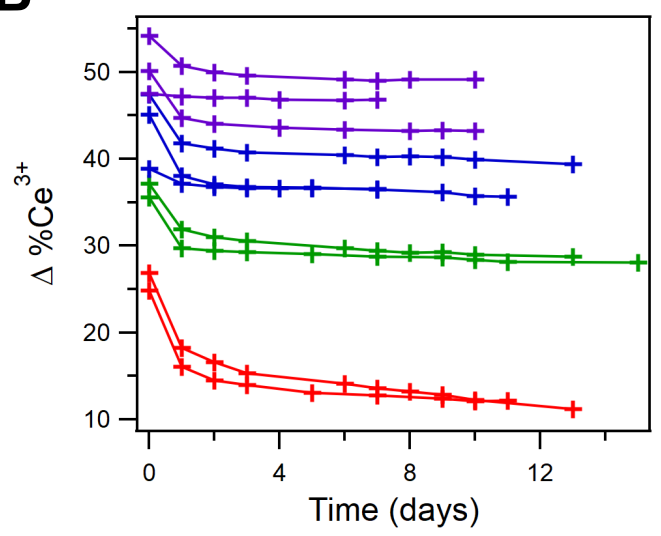

D

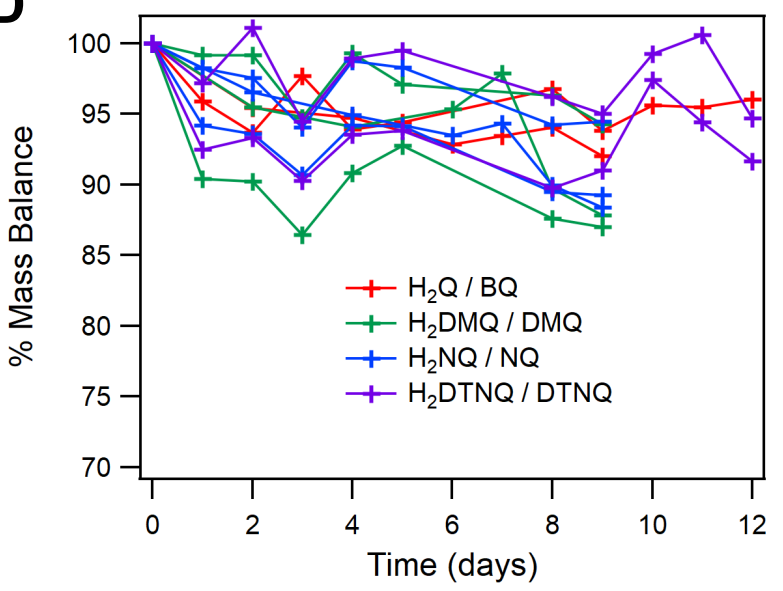

Figure S24. Time courses between OLE-Ce colloids and $\mathrm{H}_{2} \mathrm{BQ} / \mathrm{BQ}$ (red), $\mathrm{H}_{2} \mathrm{DMQ} / \mathrm{DMQ}$ (green), $\mathrm{H}_{2} \mathrm{NQ} / \mathrm{NQ}$ (blue), and $\mathrm{H}_{2} \mathrm{DTNQ} / \mathrm{DTNQ}$ (purple) by ${ }^{1} \mathrm{H}$ NMR. Data plotted for (A) hydroquinone oxidation by Ce-1 and (B) quinone reduction by Ce-1. Additionally, time courses for (C) hydroquinone oxidation by Ce-2 and the associated (D) mass balance of hydroquinone and quinone for each run as a function of time. Values determined by ${ }^{1} \mathrm{H}$ NMR vs. a 1,3,5-trimethoxybenzene internal standard.
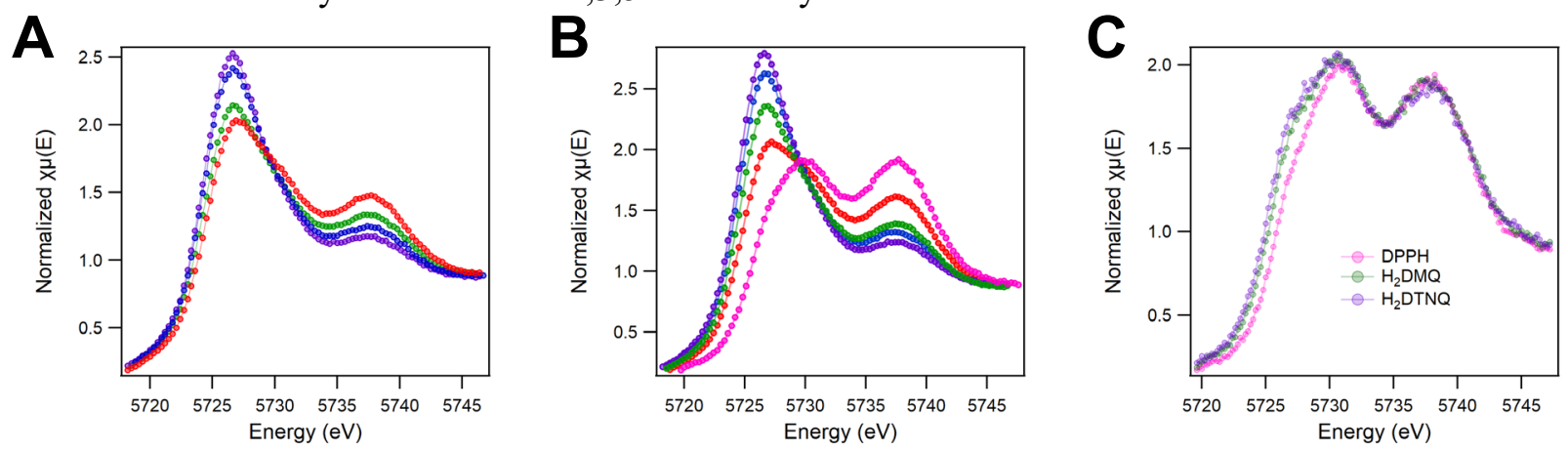

Figure S25. XANES spectra at the Ce $\mathrm{L}_{\mathrm{III}}$-edge of DPPH/DPPH-H (pink), $\mathrm{H}_{2} \mathrm{BQ} / \mathrm{BQ}$ (red), $\mathrm{H}_{2} \mathrm{DMQ} / \mathrm{DMQ}$ (green), $\mathrm{H}_{2} \mathrm{NQ} / \mathrm{NQ}$ (blue), and $\mathrm{H}_{2} \mathrm{DTNQ} / \mathrm{DTNQ}$ (purple) equilibrated with either (A) Ce-1, (B) Ce-2, or (C) Ce-L. 
Table S5. ${ }^{1} \mathrm{H}$ NMR Data for Equilibrated OLE-Ce Samples. ${ }^{a}$

\begin{tabular}{|c|c|c|c|c|c|}
\hline Reagent & $\mathbf{X H}_{n}$ & $\mathbf{X}$ & $\Delta \% \mathrm{Ce}^{3+}$ & BDFE $_{C e}$ & $E$ vs. $H_{2}$ \\
\hline DTNQ $^{b}$ & 0.15 & 2.01 & 49.1 & 62.3 & 0.45 \\
\hline $\mathrm{H}_{2} \mathrm{DTNQ}^{c}$ & 2.70 & 1.42 & 48.7 & 61.3 & 0.40 \\
\hline $\mathrm{H}_{2} \mathrm{DTNQ}^{c}$ & 1.40 & 1.42 & 48.7 & 61.5 & 0.41 \\
\hline DTNQ $^{b}$ & 0.02 & 0.87 & 46.8 & 62.6 & 0.46 \\
\hline DTNQ $^{b}$ & 0.20 & 2.16 & 43.3 & 62.2 & 0.44 \\
\hline $\mathrm{NQ}^{b}$ & 0.22 & 2.17 & 40.1 & 63.4 & 0.49 \\
\hline $\mathrm{H}_{2} \mathrm{NQ}^{c}$ & 2.20 & 1.11 & 38.7 & 62.5 & 0.46 \\
\hline $\mathrm{H}_{2} \mathrm{NQ}^{c}$ & 0.96 & 1.13 & 38.0 & 62.7 & 0.46 \\
\hline $\mathrm{NQ}^{b}$ & 0.07 & 1.64 & 36.6 & 63.6 & 0.50 \\
\hline $\mathrm{NQ}^{b}$ & 0.26 & 4.57 & 36.2 & 63.6 & 0.50 \\
\hline $\mathrm{H}_{2} \mathrm{DMQ}^{c}$ & 3.04 & 0.96 & 32.7 & 64.6 & 0.55 \\
\hline $\mathrm{H}_{2} \mathrm{DMQ}^{c}$ & 2.58 & 0.87 & 29.7 & 64.6 & 0.55 \\
\hline DMQ $^{b}$ & 0.24 & 2.10 & 29.1 & 65.5 & 0.59 \\
\hline $\mathrm{DMQ}^{b}$ & 0.22 & 2.53 & 28.2 & 65.6 & 0.59 \\
\hline $\mathrm{H}_{2} \mathrm{BQ}^{c}$ & 3.28 & 0.59 & 20.1 & 66.9 & 0.65 \\
\hline $\mathrm{H}_{2} \mathrm{BQ}^{c}$ & 2.77 & 0.56 & 19.2 & 66.9 & 0.65 \\
\hline $\mathrm{BQ}^{b}$ & 0.42 & 3.64 & 12.6 & 68.0 & 0.69 \\
\hline $\mathrm{BQ}^{b}$ & 0.36 & 5.62 & 12.5 & 68.2 & 0.70 \\
\hline $\mathrm{DPPH}^{c}$ & 0.67 & 2.37 & -5.5 & 74.2 & 0.96 \\
\hline $\mathrm{DPPH}^{c}$ & 0.60 & 2.19 & -6.0 & 74.3 & 0.97 \\
\hline $\mathrm{H}_{2} \mathrm{DTNQ}^{d}$ & 1.73 & 1.19 & 40.8 & 61.4 & 0.41 \\
\hline $\mathrm{H}_{2} \mathrm{DTNQ}^{d}$ & 1.66 & 1.14 & 39.4 & 61.4 & 0.41 \\
\hline $\mathrm{H}_{2} \mathrm{NQ}^{d}$ & 1.75 & 1.02 & 35.0 & 62.5 & 0.46 \\
\hline $\mathrm{H}_{2} \mathrm{NQ}^{d}$ & 1.69 & 1.00 & 34.5 & 62.5 & 0.46 \\
\hline $\mathrm{H}_{2} \mathrm{NQ}^{d}$ & 1.74 & 1.00 & 34.3 & 62.5 & 0.46 \\
\hline $\mathrm{H}_{2} \mathrm{DMQ}^{d}$ & 2.18 & 0.83 & 28.6 & 64.6 & 0.55 \\
\hline $\mathrm{H}_{2} \mathrm{DMQ}^{d}$ & 2.17 & 0.82 & 28.1 & 64.6 & 0.55 \\
\hline $\mathrm{H}_{2} \mathrm{DMQ}^{d}$ & 2.17 & 0.81 & 27.8 & 64.6 & 0.55 \\
\hline $\mathrm{H}_{2} \mathrm{BQ}^{d}$ & 2.30 & 0.56 & 19.4 & 67.0 & 0.65 \\
\hline $\mathrm{H}_{2} \mathrm{BQ}^{d}$ & 2.32 & 0.56 & 19.3 & 67.0 & 0.65 \\
\hline $\mathrm{DPPH}^{d}$ & 0.63 & 0.12 & -9.8 & 72.5 & 0.89 \\
\hline $\mathrm{DPPH}^{d}$ & 0.53 & 0.29 & -10.7 & 73.1 & 0.92 \\
\hline $\mathrm{H}_{2} \mathrm{DTNQ}^{e}$ & 2.93 & 0.24 & 9.8 & 60.7 & 0.38 \\
\hline $\mathrm{H}_{2} \mathrm{NQ}^{e}$ & 3.04 & 0.22 & 9.3 & 61.9 & 0.43 \\
\hline $\mathrm{H}_{2} \mathrm{DTNQ}^{e}$ & 2.93 & 0.24 & 9.2 & 60.8 & 0.38 \\
\hline $\mathrm{DTNQ}^{f}$ & 0.31 & 1.11 & 8.6 & 61.9 & 0.43 \\
\hline $\mathrm{H}_{2} \mathrm{NQ}^{e}$ & 3.06 & 0.20 & 7.7 & 61.9 & 0.43 \\
\hline $\mathrm{H}_{2} \mathrm{DMQ}^{e}$ & 3.39 & 0.15 & 6.8 & 64.0 & 0.52 \\
\hline $\mathrm{H}_{2} \mathrm{DMQ}^{e}$ & 3.40 & 0.14 & 5.8 & 64.0 & 0.52 \\
\hline $\mathrm{H}_{2} \mathrm{BQ}^{e}$ & 2.95 & 0.07 & 3.8 & 66.3 & 0.62 \\
\hline $\mathrm{H}_{2} \mathrm{BQ}^{e}$ & 2.94 & 0.07 & 3.3 & 66.3 & 0.62 \\
\hline $\mathrm{DPPH}^{e}$ & 0.43 & 0.38 & -2.4 & 73.4 & 0.93 \\
\hline
\end{tabular}

${ }^{a}$ Units are kcal mol ${ }^{-1}$ for $\mathrm{BDFE}_{\mathrm{Ce}}, \mathrm{V}$ vs. $E^{\circ}\left(\mathrm{H}^{+} / \mathrm{H}_{2}\right)$ for $E$ vs. $\mathrm{H}_{2}$, and $\mu$ mols for the other columns. ${ }^{b}$ Reacted with Ce-1 that was first reduced with $\mathrm{H}_{2}$ DCAQ. ${ }^{c}$ Reacted with Ce-1. ${ }^{d}$ Reacted with Ce-2. ${ }^{e}$ Reacted with Ce-L with equilibrium plateau at $\geq 24$ days. ${ }^{f}$ Reacted with $\mathbf{C e}-\mathbf{L}$ that was first reduced with $\mathrm{H}_{2} \mathrm{DCAQ}$. 
Table S6. XANES Data for OLE-Ce Samples. ${ }^{a}$

\begin{tabular}{|c|c|c|c|}
\hline Sample & $\% \mathrm{Ce}^{3+}$ & Sample & $\% \mathrm{Ce}^{3+}$ \\
\hline Ce-1 & 29.5 & Ce-1 - DPPH-H ${ }^{c}$ & 21.0 \\
\hline Ce-2 & 30.9 & Ce-1 $-m$ CPBA & 24.7 \\
\hline Ce-L & 20.5 & Ce-2 $-\mathrm{H}_{2}$ DTNQ & 65.4 \\
\hline Ce-1 $-\mathrm{H}_{2} \mathrm{DCAQ}$ & 75.9 & Ce-2 - $\mathrm{H}_{2} \mathrm{NQ}$ & 59.2 \\
\hline Ce-1 $-\mathrm{H}_{2}$ DTNQ & 65.9 & Ce-2 - DTNQ ${ }^{b}$ & 53.9 \\
\hline Ce-1 - $\mathrm{H}_{2} \mathrm{NQ}$ & 62.3 & Ce-2 - $\mathrm{H}_{2} \mathrm{DMQ}$ & 52.6 \\
\hline Ce-1 - DTNQ ${ }^{b}$ & 58.0 & Ce-2 - BQ ${ }^{b}$ & 42.4 \\
\hline Ce-1 - $\mathrm{H}_{2} \mathrm{DMQ}$ & 56.2 & Ce-2 - $\mathrm{H}_{2} \mathrm{BQ}$ & 38.7 \\
\hline Ce-1 - NQ ${ }^{b}$ & 52.6 & Ce-2 - DPPH & 15.9 \\
\hline Ce-1 - $\mathrm{H}_{2} \mathrm{DMQ}$ & 53.4 & $\mathbf{C e}-\mathbf{L}-\mathrm{H}_{2} \mathrm{DCAQ}$ & 30.4 \\
\hline Ce-1 - DMQ ${ }^{b}$ & 52.4 & $\mathbf{C e}-\mathbf{L}-\mathrm{H}_{2} \mathrm{DTNQ}$ & 19.5 \\
\hline Ce-1 - $\mathrm{H}_{2} \mathrm{BQ}$ & 43.6 & $\mathbf{C e}-\mathbf{L}-\mathrm{H}_{2} \mathrm{DMQ}$ & 16.6 \\
\hline Ce-1 $-\mathrm{BQ}^{b}$ & 37.3 & Ce-L - DPPH & 10.8 \\
\hline Ce-1 - DPPH & 22.7 & Ce-L $-m$ CPBA & 12.1 \\
\hline
\end{tabular}

${ }^{a}$ All reagents were added in excess of Ce atoms ${ }^{b}$ OLE-Ce was first reduced with $\mathrm{H}_{2}$ DCAQ before addition of organic reagent. ${ }^{c}$ Ce-1 was first oxidized with $m$ CPBA before addition of DPPH-H.

\section{BDFE Analyses}

The effect of correcting the equilibrium state data for the law of mass action is presented in Figure S26. Additionally, full fit parameters and lines of best fit to the Frumkin isotherm described in the main text (eq 8 ) are presented in Figure S27. Finally, a comparison between $\mu_{\mathrm{O}}$ from high-temperature studies of oxygen atom transfer by bulk ceria are compared to the $\mathrm{BDFE}_{\mathrm{Ce}}$ values for $\mathbf{C e - 1}$ collected in this study (Figure S28).
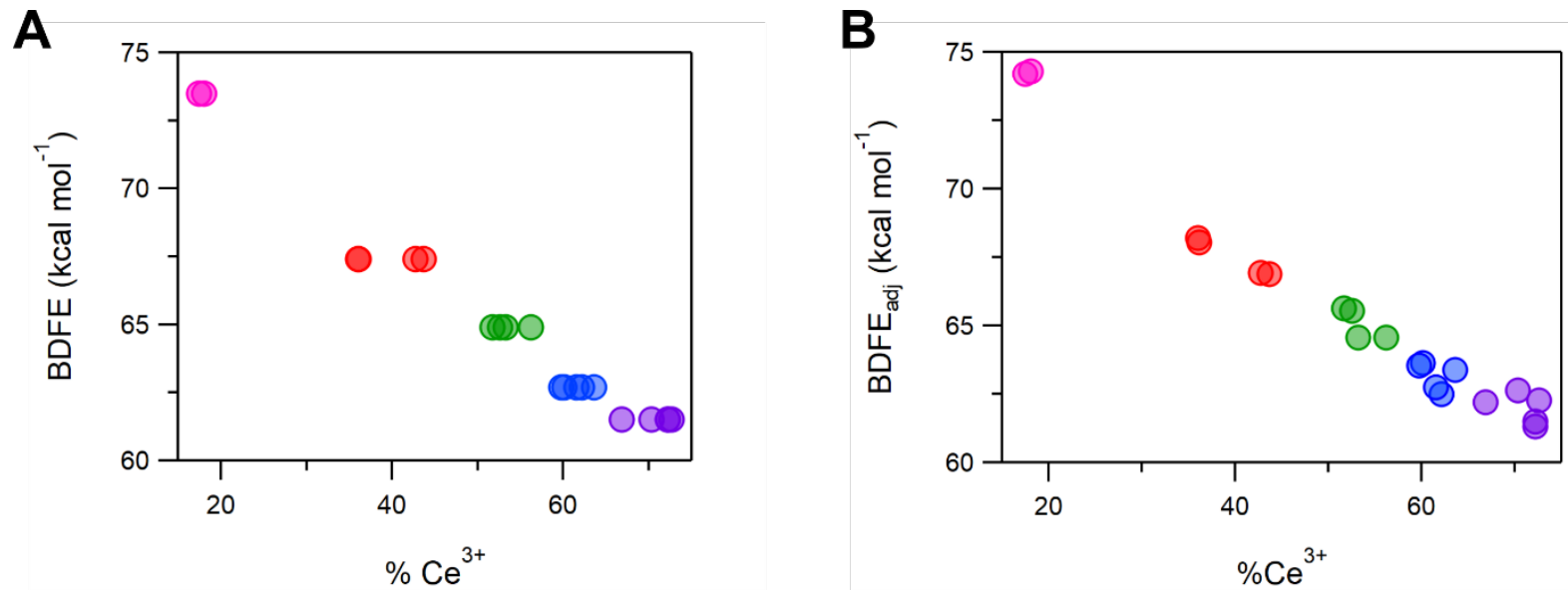

Figure S26. The $\% \mathrm{Ce}^{3+}$ at equilibrium for reactions between Ce-1 and DPPH-H/DPPH (pink), $\mathrm{H}_{2} \mathrm{BQ} / \mathrm{BQ}$ (red), $\mathrm{H}_{2} \mathrm{DMQ} / \mathrm{DMQ}$ (green), $\mathrm{H}_{2} \mathrm{NQ} / \mathrm{NQ}$ (blue), and $\mathrm{H}_{2} \mathrm{DTNQ} / \mathrm{DTNQ}$ (purple) is plotted against (A) the avg. $\mathrm{O}-\mathrm{H}$ BDFE of the hydroquinone, and (B) the adjusted avg. $\mathrm{O}-\mathrm{H}$ BDFE of the hydroquinone as determined by the Nernst equation taking into consideration the concentration of quinone and hydroquinone in solution once equilibrium was reached (eq 3 in main text). 


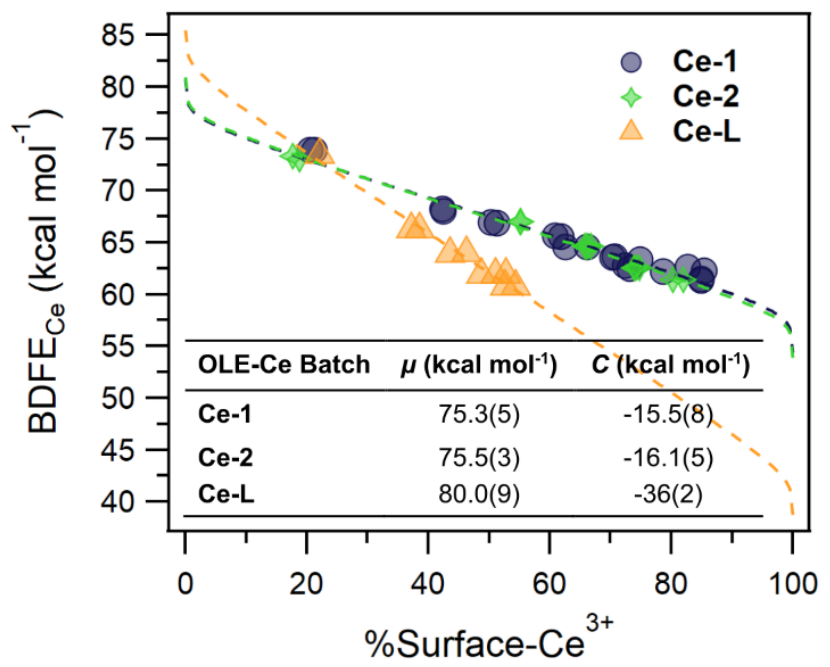

Figure S27. Plot of $\mathrm{BDFE}_{\mathrm{Ce}}$ vs. \%Surface-Ce ${ }^{3+}$ with Frumkin isotherms fits and in-set table including fit parameters from eq 8 .

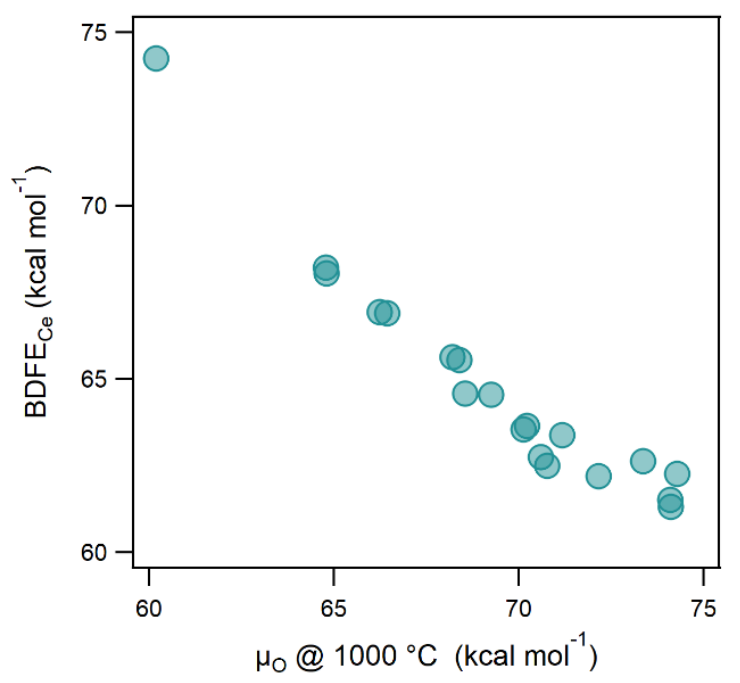

Figure S28. The quantitative relationship between the chemical potential of oxygen $\left(\mu_{\mathrm{O}}\right)$ and hydrogen $\left(\mu_{\mathrm{H}}\right)$ in ceria is estimated. $\mathrm{BDFE}_{\mathrm{Ce}}$ values for Ce-1 from Table 1 quantify $\mu_{\mathrm{H}}$ and are compared with values of $\mu_{\mathrm{O}}$ at $1,000{ }^{\circ} \mathrm{C}$ derived from a relationship between the equilibrium partial pressure of oxygen $\left(\mathrm{P}_{\mathrm{O} 2}\right)$ and oxygen vacancy concentration of bulk ceria. ${ }^{15}$ Per Bevan et al., $\mu_{\mathrm{O}}$ can be estimated as ${ }^{1} / 2 \mathrm{RT} \ln \left(\mathrm{P}_{\mathrm{O} 2}\right) .{ }^{16}$ All chemical potentials are collected at the same ratio of reduced active sites. The number of active sites is estimated as $70 \%$ of all cerium atoms for bulk ceria ${ }^{15}$ and all surface cerium atoms for Ce-1. The slope of the relationship is $-0.86(5)$, but is highly dependent on the temperature at which $\mu_{\mathrm{O}}$ is calculated, where higher temperatures increase the range of $\mu_{\mathrm{O}}$.

\section{Equilibrations under other conditions}

The effect of solvent on the equilibration of OLE-Ce with various quinones was tested by repeating equilibrations in $\mathrm{C}_{6} \mathrm{D}_{6}(\varepsilon=2.3)$ and Toluene- $\mathrm{d}_{8}(\varepsilon=2.4)$, and repeating an equilibration in THF/THF- $\mathrm{d}_{8}(\varepsilon$ $=7.6$ ) in the presence of $100 \mathrm{mM} \mathrm{TBAPF}$ (Table S7). 
The effect of additives of the equilibration of Ce-1 with $\mathrm{H}_{2}$ DMQ was also tested. Tetrabutylammonium oleate $\left(\mathrm{TBA}^{+} \mathrm{OLE}^{-}\right)$and cerium(III) oleate $\left(\mathrm{Ce}(\mathrm{OLE})_{3}\right)$ were synthesized by mixing sodium oleate with either tetrabutylammonium chloride or cerium(III) chloride in aqueous solution and then washing and isolating the insoluble product. The additions of $\mathrm{Ce}(\mathrm{OLE})_{3}$ and $\mathrm{H}_{2} \mathrm{O}$ are shown in Table S7. Additionally, time courses for the $\mathbf{C e}-1 / \mathrm{H}_{2} \mathrm{DMQ}$ equilibrium reaction in the presence of no additives, oleic acid, and $\mathrm{Ce}(\mathrm{OLE})_{3}$ are shown in

Table S7. $\% \mathrm{Ce}^{3+}$ of OLE-Ce at Equilibrium under Non-Standard Solution Conditions

\begin{tabular}{|c|c|c|c|}
\hline Sample & $\% \mathrm{Ce}^{3+}$ by ${ }^{1} \mathrm{H}$ NMR & $\% \mathrm{Ce}^{3+}$ by XANES & $\begin{array}{l}\% \mathrm{Ce}^{3+} \text { for Standard } \\
\text { Solvent Condition }\end{array}$ \\
\hline $\mathrm{H}_{2} \mathrm{BQ} / \mathbf{C e}-2+100 \mathrm{mM} \mathrm{TBAPF}_{6}{ }^{b}$ & 45 & -- & 44 \\
\hline $\mathrm{H}_{2} \mathrm{DMQ} / \mathbf{C e}-1$ in $\mathrm{C}_{6} \mathrm{D}_{6}{ }^{c}$ & 68 & 58 & 53 \\
\hline $\mathrm{H}_{2} \mathrm{DMQ} / \mathbf{C e}-2$ in Toluene- $\mathrm{d}_{8}{ }^{d}$ & 65 & -- & 53 \\
\hline $\mathrm{H}_{2} \mathrm{BQ} / \mathbf{C e}-2$ flame-sealed tube ${ }^{e}$ & 42 & -- & 44 \\
\hline $\mathrm{H}_{2} \mathrm{DMQ} / \mathbf{C e}-\mathbf{1}$ with $\mathrm{H}_{2} \mathrm{O}^{f}$ & 52 & -- & 53 \\
\hline $\mathrm{H}_{2} \mathrm{DMQ} / \mathbf{C e}-\mathbf{1}$ with $\mathrm{Ce}(\mathrm{OLE})_{3}{ }^{g}$ & 52 & -- & 53 \\
\hline
\end{tabular}

${ }^{a}$ The standard solvent condition is a mixture of THF/THF- $\mathrm{d}_{8}$ with no supporting electrolyte. ${ }^{b}[\mathrm{Ce}$ atoms] $=8.3 \mathrm{mM}$, $[\mathrm{TMB}]=8.0 \mathrm{mM}$, and $\left[\mathrm{H}_{2} \mathrm{BQ}\right]=4.7 \mathrm{mM} .^{c}[\mathrm{Ce}$ atoms $]=8.6 \mathrm{mM},[\mathrm{TMB}]=10.0 \mathrm{mM}$, and $\left[\mathrm{H}_{2} \mathrm{DMQ}\right]=5.0 \mathrm{mM} .^{d}$ $[\mathrm{Ce}$ atoms $]=8.3 \mathrm{mM},[\mathrm{TMB}]=9.0 \mathrm{mM}$, and $\left[\mathrm{H}_{2} \mathrm{DMQ}\right]=5.4 \mathrm{mM} .^{e}[$ Ce atoms $]=8.3 \mathrm{mM},[\mathrm{TMB}]=8.0 \mathrm{mM}$, and $\left[\mathrm{H}_{2} \mathrm{BQ}\right]=4.6 \mathrm{mM}^{f}[\mathrm{Ce}$ atoms $]=8.9 \mathrm{mM},[\mathrm{TMB}]=8.0 \mathrm{mM},\left[\mathrm{H}_{2} \mathrm{DMQ}\right]=4.8 \mathrm{mM}$, and $\left[\mathrm{H}_{2} \mathrm{O}\right]=49.5 \mathrm{mM} .{ }^{g}[\mathrm{Ce}$ atoms $]=8.6 \mathrm{mM},[\mathrm{TMB}]=5.8 \mathrm{mM},\left[\mathrm{H}_{2} \mathrm{DMQ}\right]=4.2 \mathrm{mM}$, and $\left[\mathrm{Ce}(\mathrm{OLE})_{3}\right]=7.0 \mathrm{mM}$.
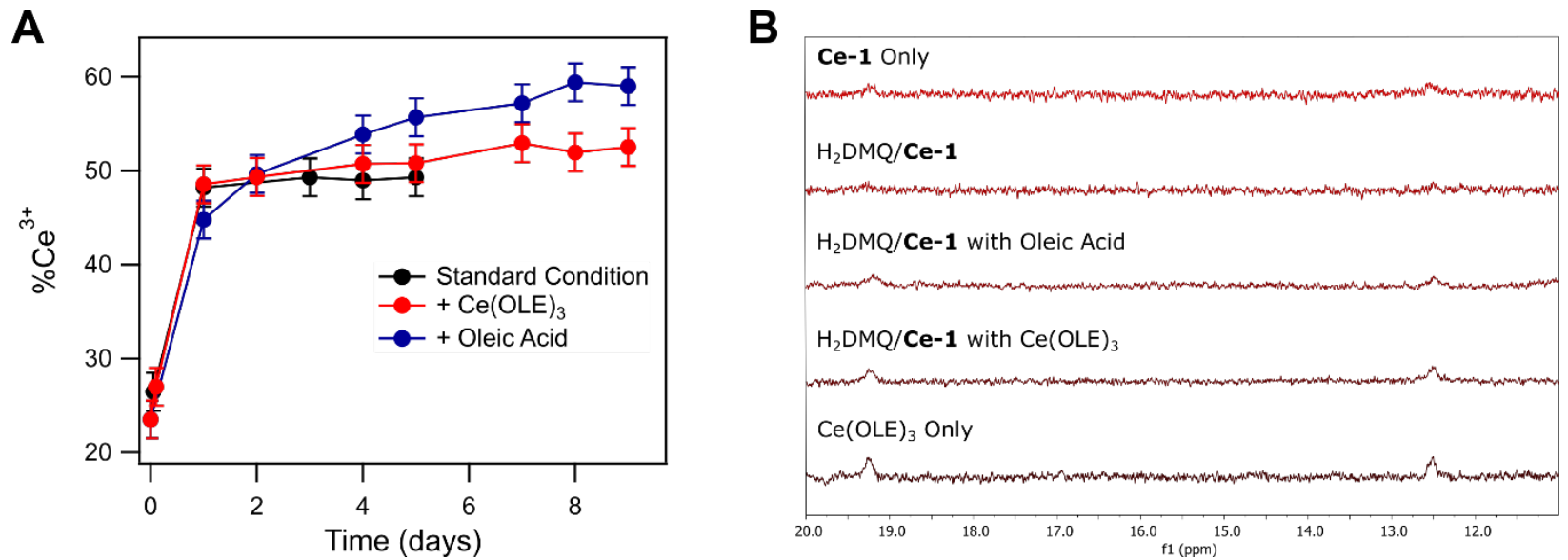

Figure S29. (A) Time courses for $\mathrm{H}_{2} \mathrm{DMQ} / \mathrm{Ce}-1$ equilibration with various additives. Standard condition contains $[\mathrm{Ce}$ atoms $]=9.1 \mathrm{mM},[\mathrm{TMB}]=6.2 \mathrm{mM}$, and $\left[\mathrm{H}_{2} \mathrm{DMQ}\right]=3.9 \mathrm{mM}$. Sample with added $\mathrm{Ce}(\mathrm{OLE})_{3}$ contains $[\mathrm{Ce}$ atoms $]=8.6 \mathrm{mM},[\mathrm{TMB}]=5.8 \mathrm{mM},\left[\mathrm{H}_{2} \mathrm{DMQ}\right]=4.2 \mathrm{mM}$, and $\left[\mathrm{Ce}(\mathrm{OLE})_{3}\right]=7.0 \mathrm{mM}$. Sample with added oleic acid (OA) contains $[\mathrm{Ce}$ atoms $]=9.0 \mathrm{mM},[\mathrm{TMB}]=6.2 \mathrm{mM},\left[\mathrm{H}_{2} \mathrm{DMQ}\right]=3.8 \mathrm{mM}$, and $[\mathrm{OA}]=24.4 \mathrm{mM}$. Error bars are estimated at $\leq 2 \% \mathrm{Ce}^{3+}$. (B) Selected sections of ${ }^{1} \mathrm{H}$ NMR spectra for same samples after reaction has been going for 5 days. 


\section{References}

(1) Taniguchi, T.; Watanabe, T.; Sakamoto, N.; Matsushita, N.; Yoshimura, M. Aqueous Route to Size-Controlled and Doped Organophilic Ceria Nanocrystals. Cryst. Growth Des. 2008, 8 (10), 3725-3730. https://doi.org/10.1021/cg800363w.

(2) Bruce, J. M. Light-Induced Reactions of Quinones. Q. Rev. Chem. Soc. 1967, 21 (3), 405. https://doi.org/10.1039/qr9672100405.

(3) Tsunekawa, S.; Ishikawa, K.; Li, Z.-Q.; Kawazoe, Y.; Kasuya, A. Origin of Anomalous Lattice Expansion in Oxide Nanoparticles. Phys. Rev. Lett. 2000, 85 (16), 3440-3443. https://doi.org/10.1103/PhysRevLett.85.3440.

(4) Wang, Z. L.; Feng, X. Polyhedral Shapes of $\mathrm{CeO}_{2}$ Nanoparticles. J. Phys. Chem. B 2003, 107 (49), 13563-13566. https://doi.org/10.1021/jp036815m.

(5) Roulhac, P. L.; Palenik, G. J. Bond Valence Sums in Coordination Chemistry. The Calculation of the Oxidation State of Cerium in Complexes Containing Cerium Bonded Only to Oxygen. Inorg. Chem. 2003, 42 (1), 118-121. https://doi.org/10.1021/ic025980y.

(6) Ravel, B.; Newville, M. ATHENA, ARTEMIS, HEPHAESTUS: Data Analysis for X-Ray Absorption Spectroscopy Using IFEFFIT. J. Synchrotron Radiat. 2005, 12 (4), 537-541. https://doi.org/10.1107/S0909049505012719.

(7) Nachimuthu, P.; Shih, W.-C.; Liu, R.-S.; Jang, L.-Y.; Chen, J.-M. The Study of Nanocrystalline Cerium Oxide by X-Ray Absorption Spectroscopy. J. Solid State Chem. 2000, 149 (2), 408-413. https://doi.org/10.1006/jssc.1999.8566.

(8) Cafun, J.-D.; Kvashnina, K. O.; Casals, E.; Puntes, V. F.; Glatzel, P. Absence of $\mathrm{Ce}^{3+}$ Sites in Chemically Active Colloidal Ceria Nanoparticles. ACS Nano 2013, 7 (12), 10726-10732. https://doi.org/10.1021/nn403542p.

(9) Zhang, J.; Wu, Z.; Liu, T.; Hu, T.; Wu, Z.; Ju, X. XANES Study on the Valence Transitions in Cerium Oxide Nanoparticles. J. Synchrotron Radiat. 2001, 8, 531. https://doi.org/10.1107/S0909049500016022.

(10) Damatov, D.; Laga, S. M.; Mader, E. A.; Peng, J.; Agarwal, R. G.; Mayer, J. M. Redox Reactivity of Colloidal Nanoceria and Use of Optical Spectra as an In Situ Monitor of Ce Oxidation States. Inorg. Chem. 2018, 57 (22), 14401-14408. https://doi.org/10.1021/acs.inorgchem.8b02598.

(11) De Vries, J. G.; Kellogg, R. M. Reduction of Aldehydes and Ketones by Sodium Dithionite. J. Org. Chem. 1980, 45 (21), 4126-4129. https://doi.org/10.1021/jo01309a011.

(12) Wise, C. F.; Agarwal, R. G.; Mayer, J. M. Determining Proton-Coupled Standard Potentials and $\mathrm{X}-\mathrm{H}$ Bond Dissociation Free Energies in Nonaqueous Solvents Using Open-Circuit Potential Measurements. J. Am. Chem. Soc. 2020, jacs.0c01032. https://doi.org/10.1021/jacs.0c01032.

(13) Carpino, L. A.; Triolo, S. A.; Berglund, R. A. Reductive Lactonization of Strategically Methylated Quinone Propionic Acid Esters and Amides. J. Org. Chem. 1989, 54 (14), 3303-3310. https://doi.org/10.1021/jo00275a012.

(14) Nakayama, T.; Nakamura, N.; Miki, S.; Hamanoue, K. Formation of Anthrahydroquinones $\left(\mathrm{XAQH}_{2}\right)$ via Excited Singlet Charge-Transfer Complexes (or Singlet Ion Pairs) of Anthraquinones with 2,5-Dimethylhexa-2,4-Diene (DMHD), and Photochemical Reaction of $\mathrm{XAQH}_{2}$ with DMHD. J. Chem. Soc. Faraday Trans. 1995, 91 (4), 607. https://doi.org/10.1039/ft9959100607. 
(15) Bulfin, B.; Lowe, A. J.; Keogh, K. A.; Murphy, B. E.; Lübben, O.; Krasnikov, S. A.; Shvets, I. V. Analytical Model of $\mathrm{CeO}_{2}$ Oxidation and Reduction. J. Phys. Chem. C 2013, 117 (46), 24129-24137. https://doi.org/10.1021/jp406578z.

(16) Bevan, D. J. M.; Kordis, J. Mixed Oxides of the Type $\mathrm{MO}_{2}$ (Fluorite) $-\mathrm{M}_{2} \mathrm{O}_{3}-\mathrm{I}$ Oxygen Dissociation Pressures and Phase Relationships in the System $\mathrm{CeO}_{2} \square \mathrm{Ce}_{2} \mathrm{O}_{3}$ at High Temperatures. J. Inorg. Nucl. Chem. 1964, 26 (9), 1509-1523. https://doi.org/10.1016/0022-1902(64)80038-5. 